\title{
Nonlinear effects in a discrete-time dynamic model of a stock market
}

\author{
Gian Italo Bischi \\ Istituto di Scienze Economiche, University of Urbino, 61029 URBINO (Italy) \\ e-mailbischi@econ.uiurb.it \\ Vincenzo Valori \\ DIMADEFAS, University of Firenze, 50134 Firenze, Italy \\ e-mailvalori@posta.cce.unifi.it
}

\begin{abstract}
The time evolution of prices and savings in a stock market is modeled by a discrete time nonlinear dynamical system. The model proposed has a unique and unstable steady-state, so that the time evolution is determined by the nonlinear effects acting out of the equilibrium. The nonlinearities strongly influence the kind of long-run dynamics of the system. In particular, the global geometric properties of the noninvertible map of the plane, whose iteration gives the evolution of the system, are important to understand the global bifurcations which change the qualitative properties of the asymptotic dynamics. Such global bifurcations are studied by geometric and numerical methods based on the theory of critical curves, a powerful tool for the characterization of the global dynamical properties of noninvertible mappings of the plane. The model unfolds more complex chaotic and unpredictable trajectories as a consequence of increasing agents' "speculative" or "capital gain realizing" attitudes. The global analysis indicates that, for some ranges of the parameter values, the system has several coexisting attractors, and it may not be robust with respect to exogenous shocks due to the complexity of the basin of attraction.
\end{abstract}

\section{Introduction}

One of the cornerstones of the modern theory of finance is the view that asset prices exhibit erratic behavior. In this paper we consider a discrete time deterministic model, proposed in [10], for the description of the interactions between the price index of a stock market and the net stock of savings collected by the mutual funds, and we study the outcome of different kinds of complexity, not only related to the creation of chaotic attractors, but also to the existence of several coexisting attractors with a complex structure of the corresponding basins of attraction.

The model is based on the assumption that two different kinds of economic agents are interacting in the market: the "dealers", who are directly admitted to the securities negotiation, and the "savers" who intend to invest in the stock market but, being scarcely informed, prefer to underwrite shares of a mutual fund. We remark that, in this scheme, both the administrators of the mutual 
funds and the agents that directly hold in their portfolio the securities are qualified as dealers. It is worth to note that we do not use the term "dealer" in a proper sense. In a Dealers Market ${ }^{1}$ a special kind of agent (the dealer), holding his own portfolio, is always willing to buy or to sell each security at the indicated price, whereas in the model the dealer chooses the securities on the market and sells fractions of the whole portfolio (the shares of the mutual fund). Anyway, as far as we are concerned, such differences are not so important.

These two different classes of agents act on two different markets and the dynamical process that regulates the evolution of the system reflects such a segmentation. The dealers obtain their profits by selling the securities at a price higher then buying, and the difference between the two prices is said "bid-ask spread".

If the competition among the dealers reduces the bid-ask spread toward zero (at the limit), no transaction will be convenient outside the dealers market as, for any given price higher (resp. lower) than the dealers' one, the buyer (resp. the seller) will prefer to buy (resp. to sell) from a dealer, while for a price equal to the dealers' one no incentives are present to prefer the direct search instead of the dealers market that is more easily accessible.

So, in a dealers market with low bid-ask spreads, the greater part of the transactions will be settled through a dealer and the dealer's choices will considerably affect the prices' course (see [9] for a wide and simple introduction to the theory of securities markets).

The essence of the stock market and of the mechanisms which regulate the prices suggest a dynamic description in discrete time, where the time unit may be seen as the interval which separates two different Stock Exchange Lists.

From the point of view of the dynamical properties of the model, the fact that, as we shall see, no stable stationary states exist for economically feasible values of the parameters, indicates the necessity of a global study, because the effects of the nonlinearities become particularly important for trajectories which move far from the steady state. Moreover, the dynamic model considered is obtained by the iteration of a noninvertible map, and this gives us the opportunity to use some recent results on noninvertible maps of the plane and to put in evidence some interesting consequences of these results. In particular, the method of critical curves can be usefully applied to understand some kinds of routes towards complex behaviors which are peculiar of noninvertible maps. The critical curves are also used to put some order in the situations of chaotic behavior, in the sense that segments of critical curves are used to bound regions of the plane inside which all the long-run dynamics of the model are trapped, even if such dynamics are quite irregular.

\footnotetext{
${ }^{1}$ It is possible to distinguish among different classes of stock markets according to the fact that the agents should search by themselves for a consistent counterpart (Direct Search Markets), or the agents could apply to specialized mediators in order to carry out such a search (Brokers Markets), or the agents could strike at once their transactions with operators who are always disposed to buy or to sell (Dealers Markets), or the agents could directly negotiate by means of a sole, centralized, mediator (Auction Markets).
} 
The plan of the paper is the following. In Section 2 we describe the economic motivations and the general structure of the model, together with some general properties arising from the symmetry of the related discrete dynamical system. In Section 3 the usual analysis of the linear approximation, through the study of the eigenvalues of the Jacobian matrix, is carried out in order to characterize the local stability property and the local bifurcations in the parameters space. In Section 4 the global dynamical properties of the dynamical system are analyzed, and such properties are used to understand the nonlinear effects that cause important qualitative changes in the structure of the attracting sets and of their basins of attraction. In this section the definitions and some properties of the critical curves are recalled, and then the critical curves are used to characterize some global bifurcations which cause qualitative changes of the attractors and their basins. Some conlcluding remarks are given in Section 5.

\section{The model}

We assume the day as the time's unit of measure. This allows us to give the following simple description of the rules which regulate the time evolution price index $p$ and of the net stock of savings collected by the funds, $s$. However, it is always possible, with little changes to our story, to refer to different intervals of time.

If the price level $p_{t}$ and the savings collected by the funds $s_{t}$ at time $t$ are supposed to be common knowledge, at time $t+1$ the stock market, at whose negotiations will participate only the dealers, will open with a new value of the index $p$, determined by a law of the kind

$$
p_{t+1}-p_{t}=g\left(s_{t}, p_{t}\right)
$$

Afterwards, being closed the stock market, the savers, who act underwriting shares of the mutual fund or asking for the repayment of the already held ones, will buy or sell, and such choices give rise to the new value of the variable $s$ through a law of the kind

$$
s_{t+1}-s_{t}=f\left(s_{t}, p_{t+1}, p_{t+1}-p_{t}\right)
$$

We remark that this two-period structure of the model can be connected with the different nature of the financial operations carried out by each class of agents and also with the legislation which, in many countries, regulate the stock market working.

The two functions $g$ and $f$ are supposed to be at least $C^{1}$ and satisfying the following assumptions:

$1-\frac{d g}{d s_{t}}>0$. This follows from the previous discussion about the role of the mutual funds in supporting the demand. 
$2-\frac{d g}{d p_{t}}<0$. In presence of an high price level the supply will grow up giving rise to a price reduction.

$3-\frac{d f}{d s_{t}}<0$. The savers prefer to diversify their investments.

$4-\frac{d f}{d p_{t+1}}<0$. The savers have a certain propensity to profit of good market performances realizing their invested capitals.

$5-\frac{d f}{d\left(p_{t+1}-p_{t}\right)}>0$. The savers have adaptive expectations. If they observe a positive trend they suppose that it will be verified in the following period too. This could persuade the investors to buy new shares of the mutual funds.

Observe that the assumptions 4 and 5 force the system into different directions. They will carry out an important role in our analysis of the model.

It is easy to verify (see [2] and [10]) that the previous five hypotheses, together with the usual regularity assumptions, are sufficient to ensure the uniqueness of the equilibrium, if it exists.

Such equilibrium values, say $\bar{s}$ and $\bar{p}$, can be considered as "natural levels", which may be thought as solutions of a system of equations or deduced from some general macroeconomic considerations, that the agents perceive as reference values to which they compare the present situation in order to take the investment decision. Under these assumptions the model can be rewritten as

$$
\left\{\begin{array}{l}
s_{t+1}-s_{t}=F\left(s_{t}-\bar{s}, p_{t+1}-\bar{p}, p_{t+1}-p_{t}\right) \\
p_{t+1}-p_{t}=G\left(s_{t}-\bar{s}, p_{t}-\bar{p}\right)
\end{array}\right.
$$

whose dynamics depend on the differences from the values that the agents perceive as natural.

The functions $F$ and $G$ satisfy the same first order conditions defined for $f$ and $g$, but in this context it is natural to claim that

$$
G(0,0)=F(0,0,0)=0
$$

After the following change of variables

$$
S_{t}=s_{t}-\bar{s} \quad \text { and } \quad P_{t}=p_{t}-\bar{p}
$$

we obtain the model

$$
\left\{\begin{array}{l}
S_{t+1}-S_{t}=F\left(S_{t}, P_{t+1}, P_{t+1}-P_{t}\right) \\
P_{t+1}-P_{t}=G\left(S_{t}, P_{t}\right)
\end{array}\right.
$$

whose only equilibrium is $O=(0,0)$.

Following the approach of [2] and [10], as a consequence of some further assumptions about the prevailing behavior of the agents, we specify the map in a polynomial form introducing near the linear terms a stabilizing component of the third order as follows: 


$$
\left\{\begin{array}{l}
S_{t+1}-S_{t}=-A P_{t+1}-B S_{t}^{3}+E\left(P_{t+1}-P_{t}\right) \\
P_{t+1}-P_{t}=C S_{t}-D P_{t}^{3}
\end{array}\right.
$$

where all the coefficients, $A, B, C, D, E$, whose meaning can be easily deduced from the previous discussion about the assumptions $1, \ldots, 5$, are real and positive.

Near the equilibrium, the dynamics of each variable are mainly influenced by the value of the other one, while the opposite occurs when the system is far from the equilibrium. In fact, we may think that the decision of the savers in order to rebalance their portfolios become significant only when the difference $s_{t}-\bar{s}$ is big. At the same time, in the stock market the supply, involving also the new securities issue, is thought to be poorly reactive with respect to the difference $p_{t}-\bar{p}$ near the equilibrium.

To sum up, the time evolution of the system is obtained by the iteration of the two-dimensional $\operatorname{map} T:(S, P) \rightarrow\left(S^{\prime}, P^{\prime}\right)$, defined by

$$
T:\left\{\begin{array}{l}
S^{\prime}=(1+e c) S-a P-b S^{3}-e d P^{3} \\
P^{\prime}=c S+P-d P^{3}
\end{array}\right.
$$

where ' denotes the unit-time advancement operator, that is, if the right hand side variables represent the dynamic variables at period $t$ then the left hand side ones represent the state variables at period $(t+1)$, and

$$
a=A, b=B, c=C, d=D, e=E-A
$$

According to the above discussion, the parameters $a, b, c$ and $d$ are positive, whereas the coefficient $e$ can take negative values provided that

$$
e+a>0
$$

Starting from a given initial condition

$$
(S(0), P(0))=\left(S_{0}, P_{0}\right)
$$

the iteration of (6) uniquely determines an infinite sequence of points, or trajectory

$$
\tau\left(S_{0}, P_{0}\right)=\left\{(S(t), P(t))=T^{t}\left(S_{0}, P_{0}\right), t=0,1,2, \ldots\right\} .
$$

Our goal is to investigate, for any economically feasible set of parameters, the possible kinds of asymptotic (or long-run) dynamics of the model proposed, i.e. the fate of the trajectories as $t \rightarrow+\infty$, and the influence of the initial conditions on it. 
It is worth noting that the map $T$ is symmetric with respect to the fixed point $O$. In fact, if $S I M:(S, P) \rightarrow(-P,-S)$ denotes the central symmetry with respect to the point $O=(0,0)$, then we have that $T$ commutes with $S I M$, i.e.

$$
T(\operatorname{SIM}(S, P))=\operatorname{SIM}(T(S, P)) .
$$

This implies that given an orbit $G$ of $T$, either $S I M(G)=G$, i.e. $G$ it is symmetric with respect to $O$, or $\operatorname{SIM}(G) \neq G$ is an orbit of $T$ as well, where $\operatorname{SIM}(G)$ is the orbit symmetric of $G$ with respect to $O$. For example, if $G$ is a cycle of period $k$, say $G=C_{k}=\left\{\left(S_{1}, P_{1}\right), \ldots,\left(S_{k}, P_{k}\right)\right\}$, then either $C_{k}$ is symmetric with respect to $O$, i.e. for each periodic point $\left(S_{i}, P_{i}\right) \in C_{k}$ also $\operatorname{SIM}\left(S_{i}, P_{i}\right)=\left(-S_{i},-P_{i}\right) \in C_{k}$, or the symmetric $k$-cycle $C_{k}^{\prime}=\left\{\left(-S_{1},-P_{1}\right), \ldots,\left(-S_{k},-P_{k}\right)\right\}$ exist, with the same stability property. This implies, in particular, that if $C_{2 j+1}$ is a cycle of odd period, then a distinct cycle $C_{2 j+1}^{\prime}=S I M\left(C_{2 j+1}\right)$ exists with periodic points which are symmetric of those of $C_{2 j+1}$ with respect to $O$. Moreover, if $C_{2 j+1}$ is stable, $C_{2 j+1}^{\prime}$ is stable as well.

Thus the symmetry property of the map $T$ naturally leads to many situations of coexisting stable symmetric cycles. However, we shall see that also coexistence of non symmetric stable cycles of different periods can be obtained, as well as coexistence of stable cycles with quasi periodic or chaotic attractors.

\section{Local bifurcations in the enlarged parameters space}

As usual, the first step to start the qualitative study of the asymptotic behavior of a dynamic model is the localization of the steady states of the dynamical system and the determination of the sets of parameters for which they are locally stable. In our case, the following result holds

Proposition 1. For each economically feasible set of parameters, the dynamical system obtained by the iteration of (6) has the unique steady state $O=(0,0)$ which is unstable.

Proof. The steady states are the fixed points of the map (6), defined by the equation $T(S, P)=$ $(S, P)$, which reduces to

$$
\left\{\begin{array}{l}
S=\frac{d}{c} P^{3} \\
P\left(a+\frac{b d^{3}}{c^{3}} P^{8}\right)=0
\end{array} .\right.
$$

Since $a, b, c$ and $d$ are positive, the only solution of (10) is $O=(0,0)$. The local stability of the steady state $O$ is determined through the study of the linear approximation of the map (6): a sufficient condition for the local asymptotic stability of $O$ is that the eigenvalues of the Jacobian matrix

computed at $O$, i.e.

$$
D T(S, P)=\left[\begin{array}{cc}
1+e c-3 b S^{2} & -a-3 e d P^{2} \\
c & 1-3 d P^{2}
\end{array}\right]
$$

$$
D T(0,0)=\left[\begin{array}{cc}
1+e c & -a \\
c & 1
\end{array}\right]
$$


has both the eigenvalues with modulus less than one, whereas $O$ is unstable if at least one eigenvalue with modulus greater than one exists. The eigenvalues of (12), say $\lambda_{1}$ and $\lambda_{2}$, are the solutions of the characteristic equation

$$
P(\lambda)=\lambda^{2}-\operatorname{Tr} \cdot \lambda+\operatorname{Det}=0
$$

where $\operatorname{Tr}$ and $D e t$ are the trace and the determinant of (12), given by

$$
\operatorname{Tr}=2+e c \quad \text { Det }=1+c(a+e)
$$

respectively. The instability of $O$ follows from the fact that Det $=\lambda_{1} \lambda_{2}>1$ for any feasible set of the parameters.

From this proposition it follows that the time evolution of the variables $S(t)$ and $P(t)$ never settle to stationary (or equilibrium) values.

In order to understand the kinds of non stationary asymptotic dynamics of the model we now extend the analysis of the local stability of $O$ to sets of parameters which are out of the economically feasible region. We do this economic aberration because the local bifurcations of $T$ in economically meaningless regions of the parameter space may provide useful information regarding its behavior in the parameter region that we want to characterize. In the analysis which follows we shall consider fixed positive values of the parameters $b, c$ and $d$ and we investigate the effect of changes of the parameters $e \in \mathbb{R}$ and $a \in \mathbb{R}$, even if the set of their economically feasible values is given by

$$
M=\{(e, a) \mid a>0 \text { and } e+a>0\}
$$

The choice of the parameters $a$ and $e$ as bifurcation parameters is related to the fact that they give a measure of the two opposite forces which determine the relative weight of the agent's attitude to realize the capital gains, measured by the parameter $a$, and their speculative attitude, measured by the parameter $e$.

Necessary and sufficient conditions for the two solutions $\lambda_{1}$ and $\lambda_{2}$ of (13) have modulus less than one, i.e. are inside the unit circle of the complex plane, are expressed by the following system of inequalities (see, for example, [12] p. 159)

$$
P(1)=1-\operatorname{Tr}+\text { Det }>0 ; \quad P(-1)=1+\operatorname{Tr}+\text { Det }>0 ; \quad \text { Det }<1
$$

These conditions, which in our case become

$$
\begin{aligned}
& P(1)=a c>0 \\
& P(-1)=c(a+2 e)+4>0 \\
& \text { Det }-1=c(a+e)<0
\end{aligned}
$$

define a stability region in the plane $(e, a)$ represented by the triangle $L J K$ of fig.1, bounded by the lines $K L$ of equation $c(a+2 e)+4=0, L J$ of equation $a=0$ and $J K$ of equation $a+e=0$. 
INSERT FIG. 1

If the parameters $(e, a)$ exit the stability region by crossing the side $K L$ then a real eigenvalue exits the unit circle through the value -1 , and $O$ loses stability via a flip (or period doubling) bifurcation, whereas if the parameters $(e, a)$ exit the stability region by crossing the side $L J$ a real eigenvalue exits the unit circle through the value +1 , and $O$ loses stability via a pitchfork bifurcation, at which two stable fixed points are created, given by the two further real solutions of (10) existing for $a<0$. However, the most interesting bifurcation through which the fixed point $O$ loses stability is the one occurring when the parameters $(e, a)$ exit the stability region by crossing the side $J K$, because this crossing implies that the parameters enter the economically feasible region $M$. In this case two complex conjugate eigenvalues exit the unit circle so that the fixed point $O$ is transformed from a stable focus into an unstable focus. The following result holds (see [10])

Proposition 2. If $c>0$ and the parameters $(e, a)$ exit the stability region LJK through the segment $K J$ of the line of equation $a+e=0$ then a supercritical Neimark-Hopf bifurcation occurs provided that $-4 / c<e<0$ and $e c \neq\{-2,-3\}$.

Proof. The eigenvalues $\lambda_{1}$ and $\lambda_{2}$ of (12) are complex conjugate if $\operatorname{Tr}^{2}-4 D e t<0$, i.e. in the region of the parameters' plane $(e, a)$ inside the parabola $4 a-c e^{2}=0$ (see fig. 1). In this case, they are given by

$$
\lambda_{1}=\bar{\lambda}_{2}=\frac{2+e c+i \sqrt{4 a c-e^{2} c^{2}}}{2}
$$

and their modulus is $\left|\lambda_{i}\right|=\sqrt{D e t}=\sqrt{1+c(a+e)}$. If the parameters $(e, a)$ exit the stability region $L J K$ through a point $H_{0}=\left(e_{0},-e_{0}\right)$, with $-4<e_{0}<0$, then the two eigenvalues exit the unit circle, because at $H_{0}$ we have $\left|\lambda_{i}\right|=1$ and $\frac{\partial\left|\lambda_{i}\right|}{\partial e}=\frac{\partial\left|\lambda_{i}\right|}{\partial a}=c / 2>0$. Moreover, under the assumptions of the proposition, at $H_{0}$ we have $\lambda_{1}^{j} \neq 1, j=1,2,3,4$. In fact, at $H_{0}$ we have $\lambda_{1}=\frac{1}{2}\left(2+e_{0} c \pm i \sqrt{-e_{0} c\left(4+e_{0} c\right)}\right)$, so $\lambda_{1}=1$ iff $e_{0} c=0, \lambda_{1}=-1$ (so that $\left.\lambda_{1}^{2}=1\right)$ iff $e_{0} c=-2$, $\lambda_{1}=\frac{1}{2}(-1+i \sqrt{3})$ (so that $\left.\lambda_{1}^{3}=1\right)$ iff $e_{0} c=-3$ and $\lambda_{1}=i$ (so that $\lambda_{1}^{4}=1$ ) iff $e_{0} c=-4$. Hence all the assumptions for the occurrence of a Neimark-Hopf at $H_{0}$ hold (see e.g. [11]). In order to prove that such a bifurcation is supercritical it is necessary to reduce the map (6) to a normal form of the type $z^{\prime}=\lambda z+c_{1} z^{2} \bar{z}+O\left(\left|z^{5}\right|\right)$, where $z=S+i P$ and $\lambda=\lambda_{1}$. The condition for a supercritical bifurcation is $a=\operatorname{Re}\left(c_{1}\right)<0$ (see [11]). After some routine (but rather long) algebraic manipulations (we refer to [10] for details) we get

$$
a=\operatorname{Re}\left(c_{1}\right)=\frac{3}{2} \frac{1}{e_{0} c\left(4+e_{0} c\right)}\left(b-\frac{c d}{e_{0}}\right)
$$

so that $a<0$ for $\frac{4}{c}<e_{0}<0 . \square$ 
As it is well known, just after the supercritical Neimark-Hopf bifurcation, for $a+e>0$, an attracting invariant closed curve $\Gamma$ exists around the unstable focus $O$. For parameters values close to the bifurcation values such invariant closed curve is smooth and approximately of circular shape, with radius proportional to the square root of the distance from the bifurcation curve (see e.g. [11] p.305). An example is shown in fig. 2, which shows a quasi-periodic trajectory obtained for $a=1.2$, $b=0.5, c=0.4, d=0.1$ and $e=-0.9$ and starting from an initial condition close to the fixed point $O$. In fig. 2a the numerically generated trajectory is represented in the phase plane $(S, P)$ of the dynamical system (the early iterations, representing the transient, have not been plotted) and in fig. $2 \mathrm{~b}$ the a portion of the same trajectory is represented versus time. The same quasiperiodic attractor, included in the curve $\Gamma$ created at the Neimark-Hopf bifurcation, is approached by the trajectories starting from a generic initial condition taken in the white region shown in fig.2, whereas the initial conditions taken in the grey region generate diverging trajectories.

\section{INSERT FIG. 2a,b}

The Neimark-Hopf bifurcation theorem only gives local results in the parameter space, in the sense that it says nothing about the changes in the shape, or even the existence, of the invariant curve $\Gamma$, as the parameters move away from the bifurcation curve. In our model, numerical investigations show that as the parameters $a$ and $e$ move far from the Neimark-Hopf bifurcation value, the attracting invariant closed curve $\Gamma$ first increase its amplitude and maintains a smooth shape, then it starts to change its shape which becomes more and more convoluted until it is transformed into a more complex invariant set, generally an annular chaotic attractor, as shown in fig. 3, obtained with parameters $a=1.2, b=0.5, c=0.4, d=0.1$ and $e=0.55$. Of course, forecastings are more difficult in such a situation, so it is important to understand when the shape of $\Gamma$ starts to change its shape from smooth to a convoluted. In the next section we shall explain how this phenomenon is related to the fact that the map (6) is noninvertible, and we describe it in terms of interactions between $\Gamma$ and the critical curves, a powerful tool to study the global properties of noninvertible maps of the plane.

Another important question is related to the delimitation of the boundary which separates the basins of attraction of different coexisting attractors (including the basin of the attractor at infinity, i.e. the set of initial conditions generating diverging trajectories). As the parameters of the model are changed, the basins may undergo global bifurcations which change their topological structure. For example, in the situation shown in fig.3a the set of points which generate diverging trajectories (or basin of infinity) is a non connected set, since two disjoint portions of it exist, nested inside the basin of bounded trajectories. These particular topological structures of the basins are specific to noninvertible maps, and, as we shall see in the next section, their qualitative changes are caused by 
global bifurcations involving contacts between basin boundaries and critical curves. Thus, as the parameters of the model vary, we can follow two different routes to dynamic complexity: one related to more and more complex attractors, the other one related to more and more complex structures of the basins. The former route has been much more investigated in the literature, whereas the latter one has been rather neglected, even if it is very important in applications.

Contrarily to what is suggested by a comparison of the figures 2 and 3 , these two different routes to dynamic complexity are generally independent, in the sense that complex attracting sets may have very simple basins, and simple attractors (such as stable steady states) may have very complex basin boundaries (see e.g. [4], [3], [6]).

In the next section we study both the routes to complexity after a description of the global geometric properties of the map (6) and its inverses.

INSERT FIG. 3a,b

\section{Global properties and routes to complexity}

\subsection{Preimages and critical curves}

The map $T$ defined in (6) is a noninvertible map. This means that given a point $\left(S^{\prime}, P^{\prime}\right) \in \mathbb{R}^{2}$ its rank-1 preimages (or antecedents) $T^{-1}\left(S^{\prime}, P^{\prime}\right)$ may be more than one, i.e., $T^{-1}$ is a multivalued relation. Such preimages can be computed by solving, with respect to the unknowns $S$ and $P$, the algebraic system (6), which can be written as

$$
\left\{\begin{array}{l}
S=\frac{1}{c}\left(P^{\prime}-P+d P^{3}\right) \\
b\left(d P^{3}-P+P^{\prime}\right)^{3}-c^{2}\left(d P^{3}-P+P^{\prime}\right)-c^{3} e\left(P^{\prime}-P\right)+c^{3}\left(a P-S^{\prime}\right)=0
\end{array}\right.
$$

This is a ninth degree algebraic system, so it may have $1,3,5,7$, or 9 solutions, according to the values of $S^{\prime}$ and $P^{\prime}$. The plane can be subdivided into regions whose points have the same number of rank-1 preimages. Following the terminology of [13], we denote by $Z_{k}$ a region whose points have $k$ distinct preimages.

As the point $\left(S^{\prime}, P^{\prime}\right)$ varies in the plane $\mathbb{R}^{2}$, the number of solutions of the system (17), i.e., the number of the rank-one preimages of $\left(S^{\prime}, P^{\prime}\right)$, changes when the point $\left(S^{\prime}, P^{\prime}\right)$ crosses the boundary separating two different regions $Z_{k}$ because pairs of real solutions of (17) appear or disappear. Accordingly, such boundaries are generally characterized by the presence of two coincident (merging) preimages. This leads us to the definition of critical curves, one of the distinguishing features of noninvertible maps. Following [12], [13], [1], the critical set LC (from the French "Ligne Critique") is defined as the locus of points having two, or more, coincident rank-1 preimages, located on a set (set of merging preimages) called $L C_{-1}$. $L C$ is the two-dimensional generalization of the notion of 
local minimum or maximum value of a one-dimensional map ${ }^{2}, L C_{-1}$ is the generalization of the notion of critical point (when it is a local extremum point). Arcs of $L C$ separate the regions of the plane characterized by a different number of real rank-1 preimages (see [12], [13], [1],).

As in the case of differentiable one-dimensional maps, where their first derivative necessarily vanishes at the local extremum points, for a two-dimensional continuously differentiable map the set $L C_{-1}$ belongs to the set of points in which the Jacobian determinant vanishes:

$$
L C_{-1} \subseteq J_{0}=\left\{(S, P) \in \mathbb{R}^{2} \mid \operatorname{det} D T=0\right\}
$$

In fact, as $L C_{-1}$ is defined as the locus of coincident rank-1 preimages of the points of $L C$, in any neighborhood of a point of $L C_{-1}$ there are at least two distinct points mapped by $T$ in the same point near $L C$. This means that the map $T$ is not locally invertible in the points of $L C_{-1}$ and, if the map $T$ is continuously differentiable, it follows that the Jacobian necessarily vanishes along $L C_{-1}$. If the set $L C_{-1}$ is determined by (18) then $L C$ is simply obtained as the image of $L C_{-1}$, i.e., $L C=T\left(L C_{-1}\right)$.

For the polynomial map (6) $L C_{-1}$ coincides with the set of points at which $\operatorname{det} D T=0$, given by the solutions of the equation

$$
9 b d S^{2} P^{2}-3 d P^{2}-3 b S^{2}+c(a+e)+1=0
$$

Thus $L C_{-1}$ is formed by four branches, denoted by $L C_{-1}^{(j)}, j=1, \ldots, 4$ in fig.4a, located symmetrically with respect to the coordinate axes, of equations

$$
P= \pm \frac{1}{\sqrt{3 d}} \sqrt{1-\frac{c(a+e)}{3 b S^{2}-1}} \text { and } S= \pm \frac{1}{\sqrt{3 b}} \sqrt{1-\frac{c(a+e)}{3 d P^{2}-1}}
$$

It follows that also $L C=T\left(L C_{-1}\right)$ is the union of four branches, say $L C^{(j)}=T\left(L C_{-1}^{(j)}\right), j=1, \ldots, 4$, and each portion of the critical set $L C$ separates two regions $Z_{k}$ and $Z_{k+2}$ (see fig.4b and fig.5). The number of regions $Z_{k}$ and the maximum value of $k$ (i.e. the maximum number of preimages, called map degree) depend on the values of the parameters. The simplest situation occurs when the four branches of $L C$ do not intersect (like in fig. $4 \mathrm{~b}$ ): in this case, the map is of third degree, and each branch of $L C$ encloses a $Z_{3}$ region surrounded by $Z_{1}$ (a structure denoted by $Z_{3}>Z_{1}$ in [13], where the notation is related to presence of a cusp point). The points belonging to the smooth arcs of $L C^{(j)}$ have two coincident rank-1 preimages which are located at a point of the corresponding $L C_{-1}^{(j)}$, and a further rank-1 preimage, called extra preimage, located elsewhere. The cusp points are characterized by three merging preimages, i.e. also the extra-preimage merges with the two coincident rank-1 preimages (see e.g. [15]).

\footnotetext{
${ }^{2}$ This terminology, and notation, originates from the notion of critical points as it is used in the classical works of Julia and Fatou.
} 
In order to give a geometrical interpretation of the action of the multivalued inverse relation $T^{-1}$ it is useful to consider a region $Z_{k}$ as the superposition of $k$ sheets, each associated with a different inverse map. Such a representation is known as Riemann foliation of the plane (see e.g. [15], [13]). Different sheets are connected by folds joining two sheets, and the projections of such folds on the phase plane are arcs of $L C$. For example, the foliation associated with the situation shown in fig. $4 \mathrm{~b}$ is qualitatively represented in fig. 4c.

Each of the $k$ superposed sheets used to represent a region $Z_{k}$ is associated with a different inverse map, and each pair of superposed sheets which join along a fold (associated with a segment $\left.L C^{(j)}\right)$ are "unfolded" back by the action of the two corresponding inverses, say $T_{j, 1}^{-1}$ and $T_{j, 2}^{-1}$, in the sense that points of the two sheets have preimages located at opposite sides with respect to $L C_{-1}^{(j)}$. It can be noticed that each cusp point of $L C$ is characterized by the merging of three sheets at the junction of two folds.

\section{INSERT FIG. 4a,b}

As the parameters of the map (6) are changed, regions $Z_{k}$ with $k>3$ are created at the intersections of the branches of $L C$, as shown in the figures 5 and 6 .

The representation of the Riemann foliation, which gives a visualization of the property of mapping distinct points into the same point as due to a folding of the phase plane along the critical curves, may be useful in order to understand some of the properties and bifurcations observed in the attractors and the basins of dynamical systems obtained by the iteration of noninvertible maps.

\section{INSERT FIGURES 5,6}

\subsection{From attracting closed invariant curves to chaotic attractors.}

We now describe the changes of the stable invariant closed curve $\Gamma$, as the parameters $e$ and $a$ are moved away from the Neimark-Hopf bifurcation curve at which $\Gamma$ is created, due to interactions between $\Gamma$ and $L C_{-1}$. It is important to notice that just after its creation $\Gamma$ cannot be too close to $L C_{-1}$, because at the Neimark-Hopf bifurcation the eigenvalues are complex conjugate and belong to the unit circle of the complex plane, whereas along $L C_{-1}$ one eigenvalues must necessarily be zero being $\operatorname{det}(D T)=0$. Therefore, interactions between $\Gamma$ and $L C_{-1}$ are only possible when the parameters are sufficiently far from the Neimark-Hopf bifurcation curve.

As far as the attracting invariant closed curve $\Gamma$ does not intersect $L C_{-1}$ it can be thought of as entirely contained in one sheet of the Riemann foliation. This means that a neighborhood $U(\Gamma)$ 
of $\Gamma$ exists such that not only $T(U) \subset U$ (since $\Gamma$ is attracting) but a unique inverse exists, say $T_{1}^{-1}$, such that $T_{1}^{-1}: T(U) \rightarrow U$. This implies that the curve $\Gamma$, as well as the area of the phase plane enclosed by $\Gamma$, say $a(\Gamma)$, is both forward invariant (under $T$ ) and backward invariant (under $T_{1}^{-1}$ ). In fact, even if $\Gamma \in Z_{k}$ with $k>1$, so that other (extra) rank-1 preimages of $\Gamma$ exist, they do not intersect $\Gamma$.

This is the situation shown in fig.7a, obtained for $a=1.2, b=0.5, c=0.4, d=0.5$ and $e=-0.6$. In this case $\Gamma \in Z_{5}$ (compare fig.7a with fig.5) and five disjoint preimages of $\Gamma$ exist, as shown in fig. 7a. Notice that for this set of parameters the invariant curve $\Gamma$ appears to be smooth and of approximately circular shape, so that the quasi-periodic motion along it is very similar to purely trigonometric oscillations.

The situation changes when $\Gamma$ grows up until it has a contact with the two branches $L C_{-1}^{(3)}$ and $L C_{-1}^{(4)}$ of the set of merging preimages $L C_{-1}$, and then intersect them, as shown in fig. $7 \mathrm{~b}$. We now describe the consequences of the contact between $\Gamma$ and $L C_{-1}^{(4)}$. Of course, due to the symmetry property of the map (6), the same description applies to the symmetric contact between $\Gamma$ and $L C_{-1}^{(3)}$.

Let $A_{0}$ and $B_{0}$ be the two points of intersection between $\Gamma$ and $L C_{-1}^{(4)}$, and let $R_{1}$ and $R_{2}$ the two regions, separated by $L C_{-1}^{(4)}$, where there are, respectively, the codomains of the two inverses $T_{1}^{-1}$ and $T_{2}^{-1}$. Then the points $A_{1}=T\left(A_{0}\right)$ and $B_{1}=T\left(B_{0}\right)$, which must belong both to $\Gamma$ and to $L C^{(4)}=T\left(L C_{-1}^{(4)}\right)$, are points of tangential contact between $\Gamma$ and $L C^{(4)}$. In fact, the arc $A_{0} B_{0} \in \Gamma \cap R_{2}$ must be mapped by $T$ in the arc $A_{1} B_{1}=T\left(A_{0} B_{0}\right)$, entirely included in the region $Z_{5}$ on one side of $L C^{(4)}$. If we look at the preimages, we realize that now there is not a unique inverse under which $\Gamma$ is backward invariant. In fact, now $T_{1}^{-1}(\Gamma)$ also includes arcs inside $\Gamma$, like the $\operatorname{arc} A_{0} B_{0}^{(1)} \in R_{1}$, whereas $A_{0} B_{0}^{(2)} \in \Gamma \cap R_{2}$ is given by $T_{2}^{-1}\left(A_{1} B_{1}\right)$.

We can say that the region $h_{1}$ between the arc $A_{1} B_{1}$ of $\Gamma$ and $L C^{(4)}$ is "unfolded" by the action of the two inverses $T_{1}^{-1}$ and $T_{2}^{-1}$ in two distinct preimages, located in the regions $R_{1}$ and $R_{2}$ respectively, represented in fig.7b by the two portions $h_{0}^{1}=T_{1}^{-1}\left(h_{1}\right)$ and $h_{0}^{2}=T_{2}^{-1}\left(h_{1}\right)$ of $a(\Gamma)$ bounded by the two arcs $A_{0} B_{0}$ inside and along $\Gamma$ respectively. In other words, the two portions $h_{0}^{1}$ and $h_{0}^{2}$ of $a(\Gamma)$ are folded by $T$ along $L C$ to cover the area $h_{1}$, which is outside $\Gamma$. This implies that $\Gamma$ the area $a(\Gamma)$ bounded by $\Gamma$ is no longer forward invariant, since some points inside $\Gamma$ are mapped outside it (like the points belonging to $h_{0}^{1}$ and $h_{0}^{2}$ ). This phenomenon of forward invariance of a closed curve together with noninvariance of the area inside it is specific to noninvertible maps, that is, it cannot be observed in invertible ones. The property of noninvariance of $a(\Gamma)$ and the creation of convolutions of $\Gamma$ are two aspects of the same mechanism, related to the fact that curves crossing $L C_{-1}$ are folded along $L C$ and are confined into the region with an higher number of preimages.

This is only the beginning of the changes in the shape of $\Gamma$, since as $e$ is further increased the convolutions become more and more pronounced (fig.7c) until the invariant curve $\Gamma$ is broken to 
become a more complex attracting set (fig.7d). In fig. $7 \mathrm{~d}$ the presence of loops can be observed, another phenomenon which is only possible with noninvertible maps. The exact mechanism through which such loops are created, and the related loss of invariance of $\Gamma$, is still an open problem, recently studied by many authors (see e.g. [13], [7] or [8] and references therein).

\section{INSERT FIG. 7a,b,c,d}

Another consequence of the intersection between $\Gamma$ and $L C_{-1}$ is that for a periodic cycle not belonging to $\Gamma$, it may happen that some of the periodic points are inside and the others are outside the invariant curve $\Gamma$. This may be observed for the map (6) for many sets of parameters. An example is shown in fig.8, obtained for $a=7.45, b=0.5, c=0.4, d=0.1$ and $e=-5.6$, where an attracting closed invariant curve, on which quasi-periodic motion is numerically observed, is coexisting with an attracting cycle of period 6 .

\section{INSERT FIG. 8}

To sum up, just after the supercritical Neimark-Hopf bifurcation the kinds of long run dynamics which characterize the long-run behavior of the bounded trajectories are characterized by convergence to quasi-periodic or periodic attractors, located along an attracting and smooth invariant closed curve $\Gamma$. Then, when it grows up, it may become rather convoluted and then disappear. It is generally replaced by annular chaotic attractors. As usual, sets of parameters are met at which stable cycles are created via a saddle-node bifurcation. The periodic points of these stable cycles may belong to $\Gamma$, or may be inside $a(\Gamma)$, or outside $a(\Gamma)$ or, if $\Gamma$ intersects $L C_{-1}$, some of the periodic points may be inside and other outside $a(\Gamma)$. Furthermore, several coexisting attractors may be simultaneously present, such as coexisting attracting cycles or quasi-periodic or chaotic attractors together with attracting cycles. In any case, segments of the critical curves $L C$, together with a suitable number of their images $L C_{i}=T(L C)$, may be used to bound a trapping region where all the attracting sets are included. Such trapping sets, also called absorbing areas in [13], act like a bounded vessel inside which the asymptotic dynamics of the bounded trajectories are ultimately confined.

In particular, this property of the critical curves allows one to obtain the boundaries of the chaotic attractors. This is now a sufficiently known property (see [13] and references therein, see also [16] for several application to economic modeling). We recall that a chaotic area $\mathcal{A}$ of the map $T$ is an invariant set of $T$, i.e. $T(\mathcal{A}) \equiv \mathcal{A}$, which includes a chaotic set. Numerically computed trajectories seem to cover the area $\mathcal{A}$. 
Consider, for example, the trajectory shown in fig.9a, which appears to be chaotic. Following [13] or [1] a practical procedure can be outlined in order to obtain the boundary of $\mathcal{A}$ (although it is difficult to give a general method). Starting from the portion of $L_{-1}$ belonging to the invariant area $\mathcal{A}$, its images $T^{k}(\gamma)$ of increasing rank are computed until a closed region is obtained. When such a region is mapped into itself, then it is an absorbing area. For the chaotic area shown in fig.9a, four images of $\gamma=\mathcal{A} \cap L C_{-1}$, given by the segments of $L C_{-1}^{(3)}$ and $L C_{-1}^{(4)}$ shown in fig. 10a, are necessary and sufficient to obtain the whole outer boundary of $\mathcal{A}$. The complete boundary of $\mathcal{A}$ is obtained by

$$
\partial \mathcal{A} \subseteq \bigcup_{k=1}^{7} T^{k}(\gamma)
$$

as shown in fig.10b. We recall that the critical sets of rank $k$ are the images of rank $k$ of $L C_{-1}$ denoted by $L C_{k-1}=T^{k}\left(L C_{-1}\right)=T^{k-1}(L C), L C_{0}$ being $L C$. Notice that in (20) the inclusion holds because portions of the critical curves of increasing rank are also inside $\mathcal{A}$, where they indicate the regions of greater density of points, i.e. the regions that are more frequently visited by the points of the generic trajectory in the invariant area $\mathcal{A}$.

We remark that the definition of an absorbing area is also useful when non chaotic attracting sets exist, like in the case of the two coexisting stable cycles shown in fig.9b, obtained after a small variation of the parameter $e$ with respect to that used in fig.9a. In fact, also in such cases the delimitation of an absorbing area by segments of critical curves is useful to obtain a region of the phase space of the dynamical system where the asymptotic dynamics will be confined, independently of the kind (and the number) of attractors.

\section{INSERT FIGURES 9a,b and 10a,b}

\subsection{Basins and their bifurcations}

As we have seen in the previous sections, points sufficiently far from the equilibrium $O=(0,0)$ generate diverging trajectories, i.e. an attractor always exist at infinite distance. We denote by $\mathcal{B}(\infty)$ the basin of infinity, defined as the set of points which generate diverging trajectories,

$$
\mathcal{B}(\infty)=\left\{(S, P) \mid\left\|T^{t}(S, P)\right\| \rightarrow+\infty \text { as } t \rightarrow+\infty\right\}
$$

We set of bounded trajectories, denoted by $\mathcal{B}$, the complementary set of $\mathcal{B}(\infty)$, i.e.

$$
\mathcal{B}=\mathbb{R}^{2} \backslash \mathcal{B}(\infty)
$$

A delimitation of the boundary $\partial \mathcal{B}(\infty)=\partial \mathcal{B}$ which separates these two sets is important in order to know the range of state variables $(S, P)$ for which the model behaves well, since only bounded trajectories can represent meaningful time evolutions of an economic system. 
The boundary $\partial \mathcal{B}=\partial \mathcal{B}(\infty)$ behaves as a repelling set for the points near it, since it acts like a watershed for the trajectories of the map $T$. Points belonging to $\partial \mathcal{B}$ are mapped into $\partial \mathcal{B}$ both under forward and backward iteration of $T$, that is, the boundary is invariant with regard to $T$ and $T^{-1}$, i.e.

$$
T(\partial \mathcal{B})=\partial \mathcal{B}, T^{-1}(\partial \mathcal{B})=\partial \mathcal{B}
$$

This implies that if an unstable fixed point or cycle belongs to $\partial \mathcal{B}$ then $\partial \mathcal{B}$ must also contain all of its preimages of any rank. Moreover, if a saddle-point, or a saddle-cycle, belongs to $\partial \mathcal{B}$, then $\partial \mathcal{B}$ must also contain the whole stable set.

We first consider a set of parameters for which $\mathcal{B}$ is a simply connected set, as shown in fig.11a. In this case, the boundary $\partial \mathcal{B}$ is formed by the closure of the stable set of a saddle cycle $S$ of period 2 , located along $\partial \mathcal{B}$, whose numerically computed periodic points $(-1.97,3.99)$ and $(1.97,-3.99)$ are symmetrically located with respect to the point $O$. One of the two invariant branches of the unstable set $W^{u}(S)$ issuing from the saddle periodic points of $S$ goes to infinity, the other one goes to the bounded attractor, which in this case is a quasi periodic attractor located on a smooth closed invariant curve. Hence the local stable set $W_{l o c}^{s}(S) \subseteq \partial \mathcal{B}$. In fact, if we consider a neighborhood of $S, W_{l o c}^{s}(S)$ is a separatrix between the trajectories which converge to a bounded attractor and those which diverge. Then $\partial \mathcal{B}$ must include the whole stable set $W^{s}(S)$, obtained by taking the preimages of any rank of $W_{l o c}^{s}(S)$

$$
W^{s}(S)=\bigcup_{k \geq 0} T^{-k}\left(W_{l o c}^{s}(S)\right)
$$

It is worth to note that also a repelling node cycle of period two belongs to the boundary, with periodic points located at the cusp points of $\partial \mathcal{B}$.

We notice that in fig.11a the critical curve $L C^{(1)}$, as well as its symmetric branch $L C^{(2)}$, is close to $\partial \mathcal{B}$, i.e. to the stable set $W^{s}(S)$. If the parameter $e$ is slightly increased a contact between $\partial \mathcal{B}$ and the two branches $L C^{(1)}$ and $L C^{(2)}$ of $L C$ occurs. After these two contacts, two portions of $\mathcal{B}(\infty)$ that before the contact bifurcation were in the region $Z_{1}$, denoted by $H_{0}$ and $K_{0}$ in fig. $11 \mathrm{~b}$, enter the regions $Z_{3}$ bounded by $L C^{(1)}$ and $L C^{(2)}$ respectively. Each of these two portions now have new pairs of preimages, unfolded at opposite sides with respect to line $L C_{-1}^{(1)}$ and $L C_{-1}^{(2)}$ respectively. Since $H_{0}$ and $K_{0}$ belong to $\mathcal{B}(\infty)$, also their preimages belong to $\mathcal{B}(\infty)$, and constitute disjoint portions of $\mathcal{B}(\infty)$ nested inside $\mathcal{B}$. These are the largest holes (or lakes following [14]) of $\mathcal{B}(\infty)$ nested inside $\mathcal{B}$, denoted by $H_{-1}$ and $K_{-1}$ in fig.11b. Since they belong to the region $Z_{1}$, they have further preimages, which are other smaller holes (or lakes) of $\mathcal{B}(\infty)$. Such preimages are smaller and smaller and accumulate on the outer boundary (we remind that the outer boundary, being a repelling set for the forward iteration of $T$, is an attracting set for the backward iteration, obtained by the repeated application of the inverses of $T$ ). To sum up, the global bifurcation just described, 
due to a contact between $W^{s}(S)$ and $L C$, transforms the basin $\mathcal{B}$ from a simply connected set into a multiply connected one (or equivalently, it transforms the basin $\mathcal{B}(\infty)$ from connected into non connected). Now the boundary $\partial \mathcal{B}$ is formed by the union of an external part and the boundaries of the infinitely many holes.

As the parameter $e$ is further increased, the critical curves $L C^{(1)}$ and $L C^{(2)}$ continue to move until a parameter value is reached at which a contact between $L C^{(1)}$ and $L C^{(2)}$ and the two periodic points of the node located at the two cusps of $\partial \mathcal{B}$ occurs (see fig.11c). This contact causes another global bifurcation at which a reunion of the holes with the immediate basin of infinity occurs, so that $\mathcal{B}(\infty)$ becomes again a connected set (and consequently $\mathcal{B}$ becomes again simply connected).

Other similar contact bifurcations occur when portions of $\mathcal{B}(\infty)$ enter $Z_{3}$ after a contact with the other two branches of $L C, L C^{(3)}$ and $L C^{(4)}$, as shown in fig.11d. This leads to the creation of other families of holes of $\mathcal{B}(\infty)$ nested inside $\mathcal{B}$ through the same mechanism described above.

\section{INSERT FIG. 11}

We remark that, even if the occurrence of the global bifurcations described above leads to more complex basin boundaries, the attracting sets have practically maintained the same kind of complexity, as it appears from a comparison of the figures 11a-d: in figures 11a-c the bounded attractor is quasi-periodic belonging to a smooth closed invariant curve, whereas in fig. 11d the attractor is an attracting cycle of period 10, with periodic points located symmetrically with respect to $O$.

More complex situations can be observed when many coexisting bounded attractors are present, whose basins share the set $\mathcal{B}$ of points generating bounded trajectories. An example is shown in fig.12, obtained for $a=1.12, b=0.5, c=0.4, d=0.5$ and $e=1.07$. For this set of parameters there are three coexisting bounded attractors: a cycle $C_{10}$ of period 10, with points pairwise located in symmetric positions with respect to $O$, i.e. $S I M\left(C_{10}\right)=C_{10}$, where $S I M$ is the symmetry with respect to $O$ defined in section 2, and two cycles of period seven, say $C_{7}$ and $\operatorname{SIM}\left(C_{7}\right)$, one with periodic points located symmetrically with respect to the periodic points of the other, as always occurs for the cycles of odd period (as stated in section 2). The respective basins of attraction are represented by different colors: white for the basin of $C_{10}$, red and green for the basins of the two symmetric cycles of period 7. From the numerical representation of fig.12, all the basins appear to be nonconnected sets, each formed by infinitely many (and extremely intermingled) disjoint portions. Such a structure of the basins is a rather usual for noninvertible maps. This can be intuitively understood on the basis of the following arguments. Let $A$ be an attractor for the iterated map $T$. This means that a neighborhood $U(A)$ exists whose points converge to $A$. Of course $U(A) \subseteq \mathcal{B}(A)$, but also the points of the phase space which are mapped inside $U$ after a 
finite number of iterations belong to $\mathcal{B}(A)$, so that the total basin of $A$ (or more briefly the basin of $A$ ) is given by

$$
\mathcal{B}(A)=\bigcup_{n=0}^{\infty} T^{-n}(U(A))
$$

where $T^{-1}(x)$ represent the set of the rank-1 preimages of $x$ (i.e. the points mapped to $x$ by $T$ ), and $T^{-n}(x)$ represent the set of the rank- $n$ preimages of $x$ (i.e. the points mapped to $x$ after $n$ repeated applications of $T$ ). If $T^{-1}$ is a noninvertible map, the total basin may be non connected because if $U(A)$, or its preimages, belong to regions $Z_{k}$ with $k>1$, the action of the distinct inverses defined in $Z_{k}$ (i.e. defined in different sheets of the Riemann foliation) may give preimages of $U(A)$ which are disjoint from $U(A)$ and far from it, due to the unfolding of the Riemann foliation under the action of the several distinct inverses.

\section{INSERT FIG. 12}

\section{Conclusions}

In this paper we have studied a deterministic model for the description of the time evolution and interactions between savings and price level in a stock market.

The model is not based on market's microstructure and optimizing behavior of the agents, but it gives a fairly general description of the main (nonlinear) interactions between two kinds of agents that we assume are acting in two different sections of the market: the dealers (administrators of mutual funds), directly admitted to the securities negotiation, and the savers who, after taking their investment decision, buy or sell shares of the mutual funds.

The model, which had been proposed in [10], has no stable steady states for economically feasible values of the parameters, so its dynamics are always characterized by oscillatory behavior, which may be periodic, or quasi-periodic or chaotic. So, even if a deterministic framework is assumed, the model considered captures the most known features of the stock markets, given by the lack of a stable stationary situation and the existence of many kinds of oscillatory (sometimes apparently erratic) behaviors.

Our study is mainly devoted to the global dynamic properties and the global bifurcations. This study gave us the opportunity to investigate some dynamic properties and bifurcations peculiar of discrete dynamical systems which are obtained by the iteration of noninvertible maps. In fact in this model the iterated map is a ninth degree noninvertible map, and some interesting results recently given in the mathematical literature on noninvertible maps of the plane, mainly based on the method of critical curves, can be usefully applied to understand the properties of attracting sets and their basins. 
The most evident attracting sets of the model considered, which are very easily revealed even by a quick numerical exploration, are obtained from the evolution of attracting orbits generated by a Neimark-Hopf bifurcation. Immediately after the bifurcation, the attracting invariant curve fully characterizes the phase space and the market adopts a regular cyclic behavior.. Subsequently, as a consequence of increasing agents' "speculative" or "capital gain realizing" attitudes, the model unfolds more complex, chaotic and unpredictable trajectories.

However, a deeper analysis reveals that many coexisting attracting sets may be present, and the structure of their basins may be rather complex, being characterized by nonconnected sets, a phenomenon that only in noninvertible maps can occur. Such analysis has shown that ranges of the parameters exist such that the system is not robust with respect to exogenous shocks. In fact the set of initial conditions which generate bounded trajectories, reveals, also for small parameter values, "holes" of $\mathcal{B}(\infty)$, i.e. bounded regions of the phase space formed by points generating diverging trajectories which are surrounded by points of the complementary set. This means that exogenous shocks moving the system towards one of such "holes" could cause the definitive drift from the equilibrium, and the intervention of some kind of market authority will be needed.

Contact bifurcations involving the critical curves have been used to characterize two different routes to complex dynamic behaviors, one related to the creation of increasingly complex attractors as the parameters are varied, the other one related to the appearance of more complex topological structures of the basins of attraction.

The critical curves have been used both to bound trapping regions (or absorbing areas) inside which the asymptotic dynamics are ultimately bounded (thus giving an estimate of the maximum amplitude of the oscillatory properties of the market whatever the kinds of long run dynamics are) and to detect contact bifurcations which cause qualitative changes in the structure of the basins of attraction.

Acknowledgments. We thank Laura Gardini for helpful comments and suggestions. This work has been performed under the auspices of CNR, Italy, and under the activity of the national research project "Nonlinear Dynamics and Stochastic Models in Economics and Finance", MURST, Italy.

\section{References}

[1] Abraham, R., L. Gardini and C. Mira, Chaos in discrete dynamical systems (a visual introduction in two dimension) Springer-Verlag, 1997.

[2] Antoci A. "La Dinamica dei Mercati Azionari: alcuni modelli", Tesi di Laurea, Facoltà di Economia e Commercio, Università di Firenze, Firenze (1989) 
[3] G.I. Bischi and M. Kopel "On the basins of multiple Nash equilibria in nonlinear duopoly games" (submitted)

[4] G.I. Bischi, L. Gardini and M. Kopel "Analysis of Global Bifurcations in a Market Share Attraction Model", Journal of Economic Dynamics and Control (in press).

[5] G.I. Bischi and L. Gardini "Role of invariant and minimal absorbing areas in chaos synchronization", Physical Review E, vol.58 n.5, pp. 5710-5719 (1998).

[6] G.I. Bischi, L. Stefanini and L. Gardini "Synchronization, intermittency and critical curves in a duopoly game" Mathematics and Computers in Simulation, vol. 44, pp. 559-585 (1998).

[7] C.E. Frouzakis, L. Gardini, I.G. Kevrekidis, G. Millerioux and C. Mira "On some properties of invariant sets of two-dimensional noninvertible maps" International Journal of Bifurcation and Chaos, 7, 1167-1194 (1997).

[8] U. Feudel, M.A. Safonova, J. Kurtis and V.S. Anishchenko "On the destruction of threedimensional tori" International Journal of Bifurcation and Chaos, 6, 1319-1332 (1996).

[9] K. Garbade, Securities Market, Mc Graw-Hill, New York (1982).

[10] F. Gori and F. Rinaldi "Comportamenti caotici e biforcazioni in un modello dinamico discreto del mercato azionario: Una nota sulle interazioni tra livello dei prezzi e raccolta netta dei fondi", Quaderni del Dipartimento di Matematica Applicata e Informatica - Università di Venezia, Venezia (1989).

[11] Guckenheimer, J. and P. Holmes. 1983. Nonlinear Oscillations, Dynamical Systems, and Bifurcations of Vector Fields, Springer-Verlag.

[12] I. Gumowski and C. Mira, Dynamique Chaotique, Cepadues Editions, Toulose (1980).

[13] C. Mira, L. Gardini, A. Barugola and J.C. Cathala, Chaotic Dynamics in Two-Dimensional Noninvertible Maps, World Scientific (1996).

[14] C. Mira, D. Fournier-Prunaret, L. Gardini, H. Kawakami and J.C. Cathala "Basin bifurcations of two-dimensional noninvertible maps: fractalization of basins", International Journal of Bifurcation and Chaos, 4, 343-381 (1994).

[15] C. Mira, J.P. Carcasses, G. Millérioux, and L. Gardini, "Plane Foliation of Two-Dimensional Noninvertible Maps", International Journal of Bifurcation and Chaos 6 (8), 1439-1462 (1996)

[16] T. Puu, Nonlinear Dynamics, 5th edition, Springer-Verlag (1999). 


\section{Figure Captions}

Fig. 1. Extended plane of the parameters $e$ and $a$. The region $M$ represent the set of economically feasible values of the parameters. The steady state $O$ is stable inside the region $L J K$.

Fig. 2. For $a=1.2, b=0.5, c=0.4, d=0.1$ and $e=-0.9$, just after the Neimark-Hopf bifurcation, a trajectory is numerically generated starting from an initial condition close to the fixed point $O$. (a) the trajectory is represented in the phase plane $(S, P)$ (the early iterations, representing the transient, have not been plotted). The white region represents the basin of attraction of $\Gamma$, the grey region represents the basin of infinity, i.e. the set of points generating diverging trajectories. (b) A portion of the same trajectory plotted in (a) is represented versus time.

Fig. 3. For $a=1.2, b=0.5, c=0.4, d=0.1$ and $e=0.55$ the generic trajectory starting from points of the white region converge to an annular chaotic attractor, as shown in (a), whereas the points of the grey region generate diverging trajectories. In (b) a portion of the trajectory shown in (a) is represented versus time.

Fig. 4. For $a=1, b=0.5, c=0.4, d=2$ and $e=2$ the maximum number of preimages is 3 . (a) Critical curves of rank-0. (b) Critical curves of rank-1, which separates regions $Z_{k}$ whose points have $k$ distinct rank-1 preimages (c) Riemann foliation corresponding to the critical curves shown in (b). Different sheets are associated with different inverses, and the critical curves $L C$ represent folds which join different sheets

Fig. 5. For $a=1, b=0.5, c=0.4, d=0.1$ and $e=0.1$ the maximum number of preimages is 5. (a) Critical curves of rank-1, which separates regions $Z_{k}$ whose points have $k$ distinct rank-1 preimages (b) Riemann foliation corresponding to the critical curves shown in (a)

Fig. 6. For $a=1, b=0.5, c=0.4, d=0.1$ and $e=-0.9$ the maximum number of preimages is 9 . (a) Critical curves of rank-1, which separates regions $Z_{k}$ whose points have $k$ distinct rank-1 preimages (b) Riemann foliation corresponding to the critical curves shown in (a)

Fig. 7. (a) Just after the Neimark-Hopf bifurcation, for $a=1.2, b=0.5, c=0.4, d=0.1$ and $e=-0.6, \Gamma \in Z_{5}$ and $\Gamma \cap L C_{-1}=\emptyset$. The five disjoint preimages of $\Gamma$ are denoted by $T_{k}^{-1}(\Gamma)$, $k=1, \ldots, 5$. (b) For $a=1.2, b=0.5, c=0.4, d=0.1$ and $e=0, \Gamma$ intersects $L C_{-1}^{(3)}$ and $L C_{-1}^{(4)}$ and convolutions appear along $\Gamma$. (c) For $e=0.4$ (the other parameters are the same as in (a) and (b)) the convolutions become more evident. (d) For $e=0.5$ the invariant curve $\Gamma$ no longer exist and it is substituted by a more complex attracting set, characterized by the presence of loops.

Fig. 8. For $a=7.45, b=0.5, c=0.4, d=0.1$ and $e=-5.6$ an attracting cycle of period 6 exists together with an attracting closed invariant curve $\Gamma$, on which quasi-periodic motion is numerically observed. Some of the periodic points, denoted by the numbers 1, 3 and 5 , are inside $\Gamma$ and the others are outside $\Gamma$. 
Fig. 9.(a) For $a=1.2, b=0.5, c=0.4, d=0.1$ and $e=1.1$ the generic bounded trajectory is attracted towards a chaotic area. (b) For $a=1.2, b=0.5, c=0.4, d=0.1$ and $e=1.2$ the bounded trajectories converge to one of the two symmetric stable cycles of period 7 whose periodic points are respectively denoted by $\{1, \ldots, 7\}$ and $\left\{1^{\prime}, \ldots, 7^{\prime}\right\}$.

Fig. 10. For the same set of parameters used to obtain fig.9a, the two portions of $L C_{-1}$ included inside the chaotic area, indicated by the arrows, are iterated in order to obtain the boundary of the chaotic area. (a) After 4 iterations the images of the two segments of $L C_{-1}$, denoted by $L C, L C_{1}$, $L C_{2}, L C_{3}$, give the outer boundary of the chaotic area. (b) After 7 iterations the whole boundary of the chaotic area is get.

Fig. 11. Changes of the structure of the basin of infinity $\mathcal{B}(\infty)$ (grey region) due to changes in the parameter $e$, the other parameters being $a=1, b=0.5, c=0.4$ and $d=0.1$. (a) For $e=-0.6$ the set $\mathcal{B}=\mathbb{R}^{2} \backslash \mathcal{B}(\infty)$, whose points generate bounded trajectories, is a simply connected set, represented by the white region. The critical curves $L C_{-1}$ and $L C$ are also represented (compare with fig.7a). (b) For $e=-0.5$, after the contacts between $L C$ and the boundary of $\mathcal{B}(\infty)$, non connected portion (holes) of $\mathcal{B}(\infty)$ appear, nested inside the white region. (c) At $e=-0.38$ a contact between $L C^{(1)}$ and $L C^{(2)}$ and the two cusps of $\partial \mathcal{B}$ causes a reunion of the holes with the immediate basin of infinity, so that $\mathcal{B}$ becomes again a simply connected set. (b) At $e=0.3$ a contact of the other two branches of $L C$ causes the creation of other families of holes of $\mathcal{B}(\infty)$.

Fig. 12. For $a=1.12, b=0.5, c=0.4, d=0.5$ and $e=1.07$ there are three coexisting bounded attractors: a cycle $C_{10}$ of period 10 and two cycles of period seven. The respective basins of attraction are represented by different colors: white for the basin of $C_{10}$, red and green for the basins of the two symmetric cycles of period 7 . The periodic points are represented by black dots. The grey region represents the basin of infinity. 


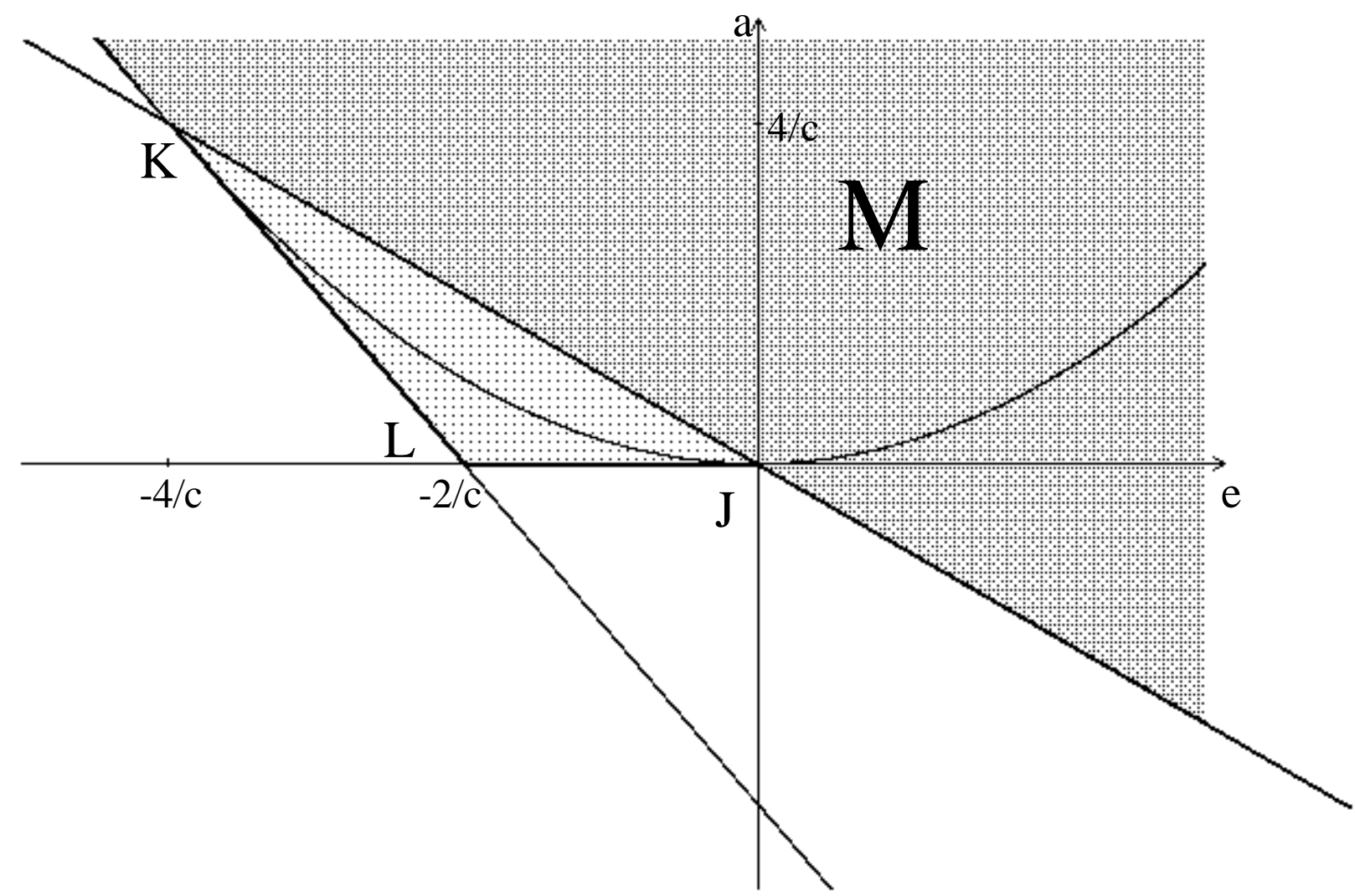

Fig. 1 


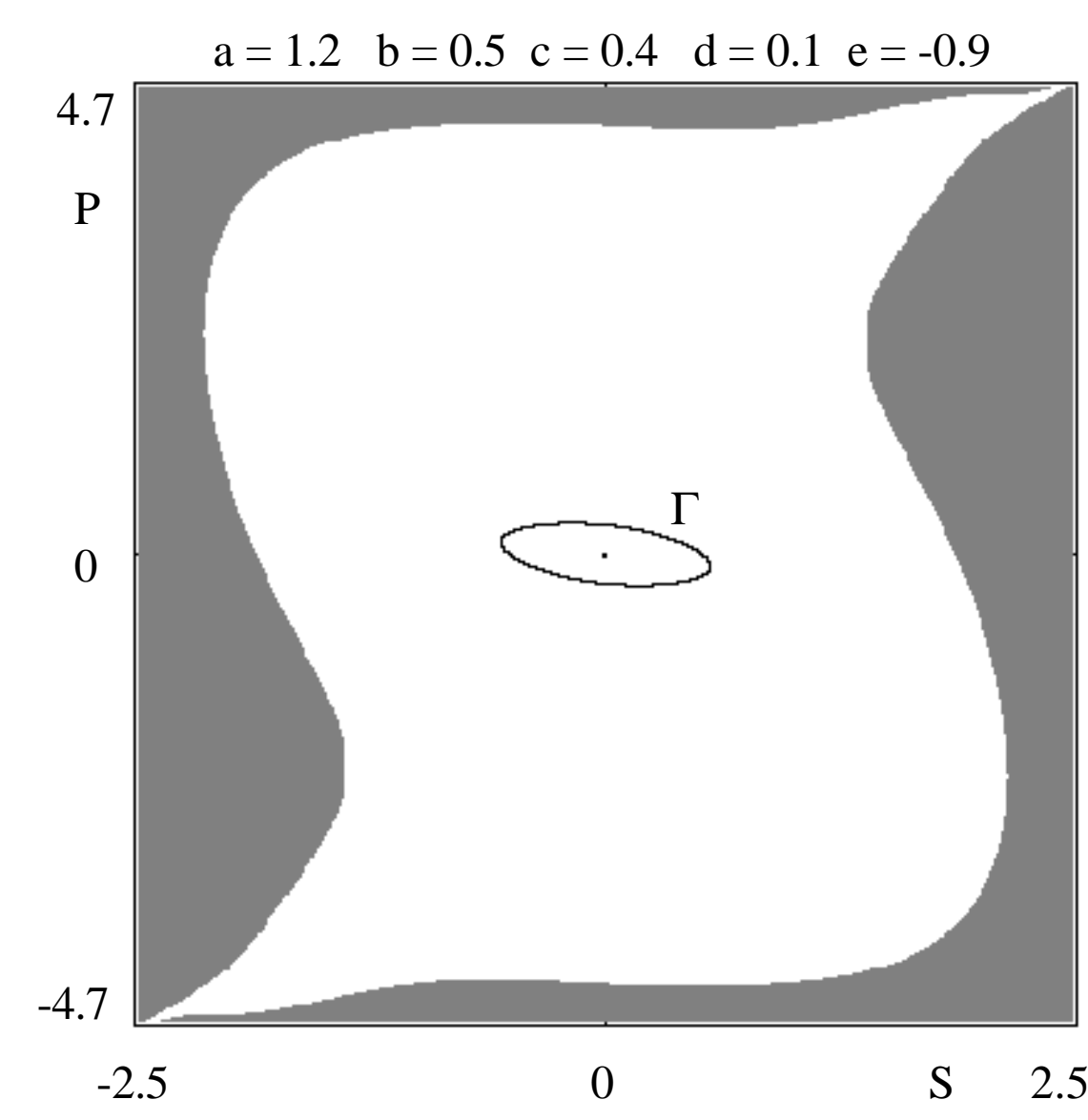

(a)
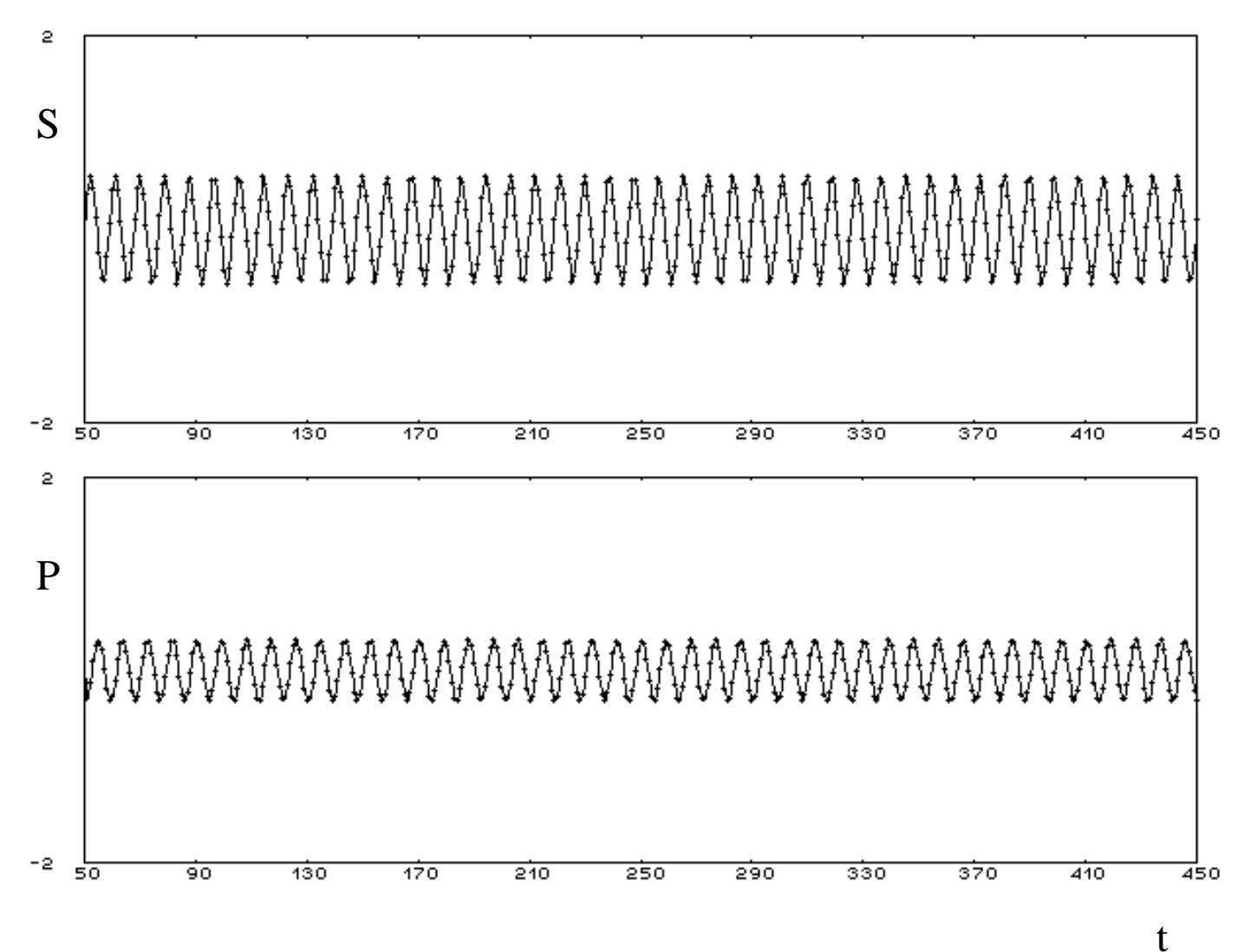

(b)

Fig. 2 


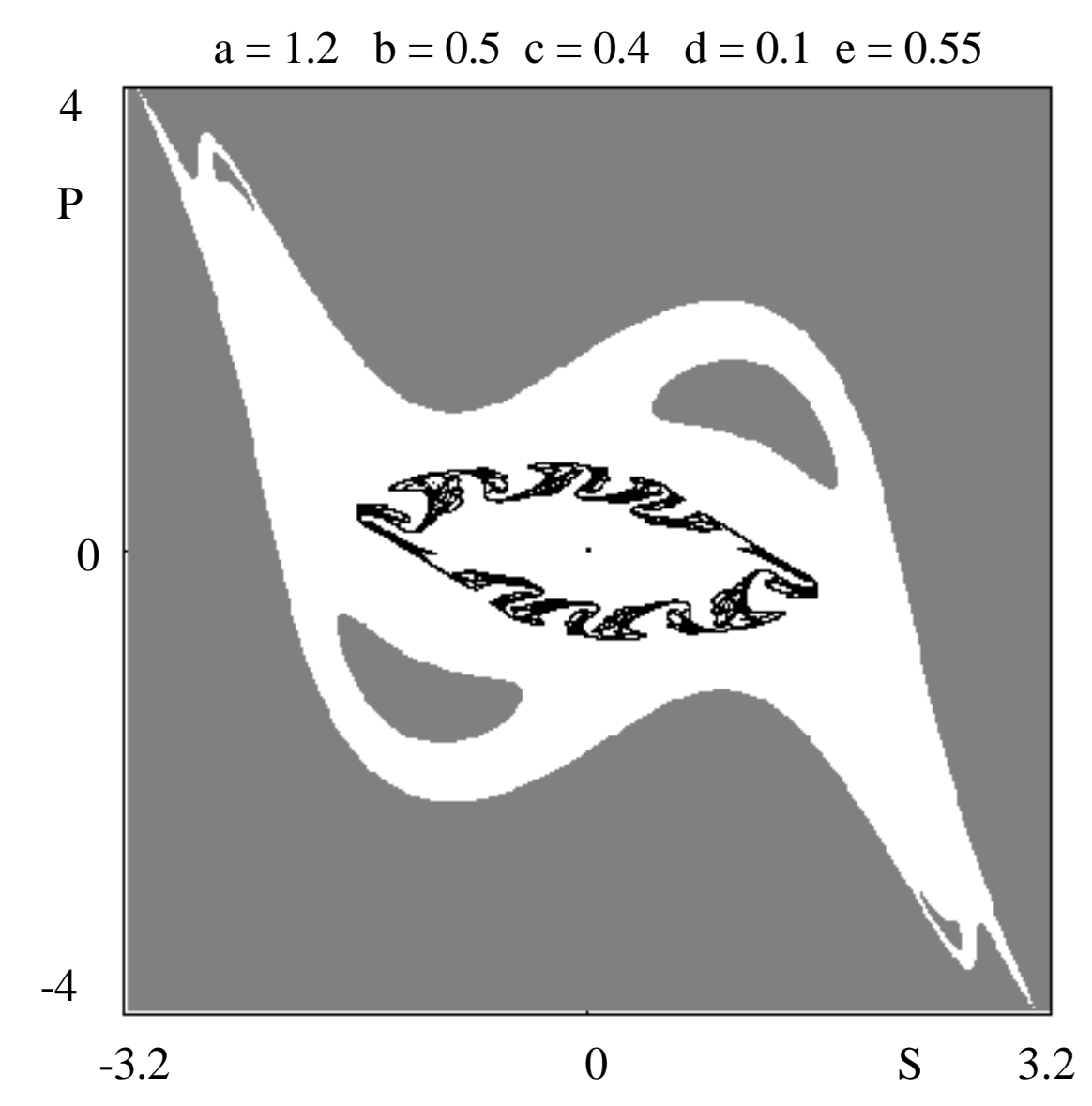

(a)
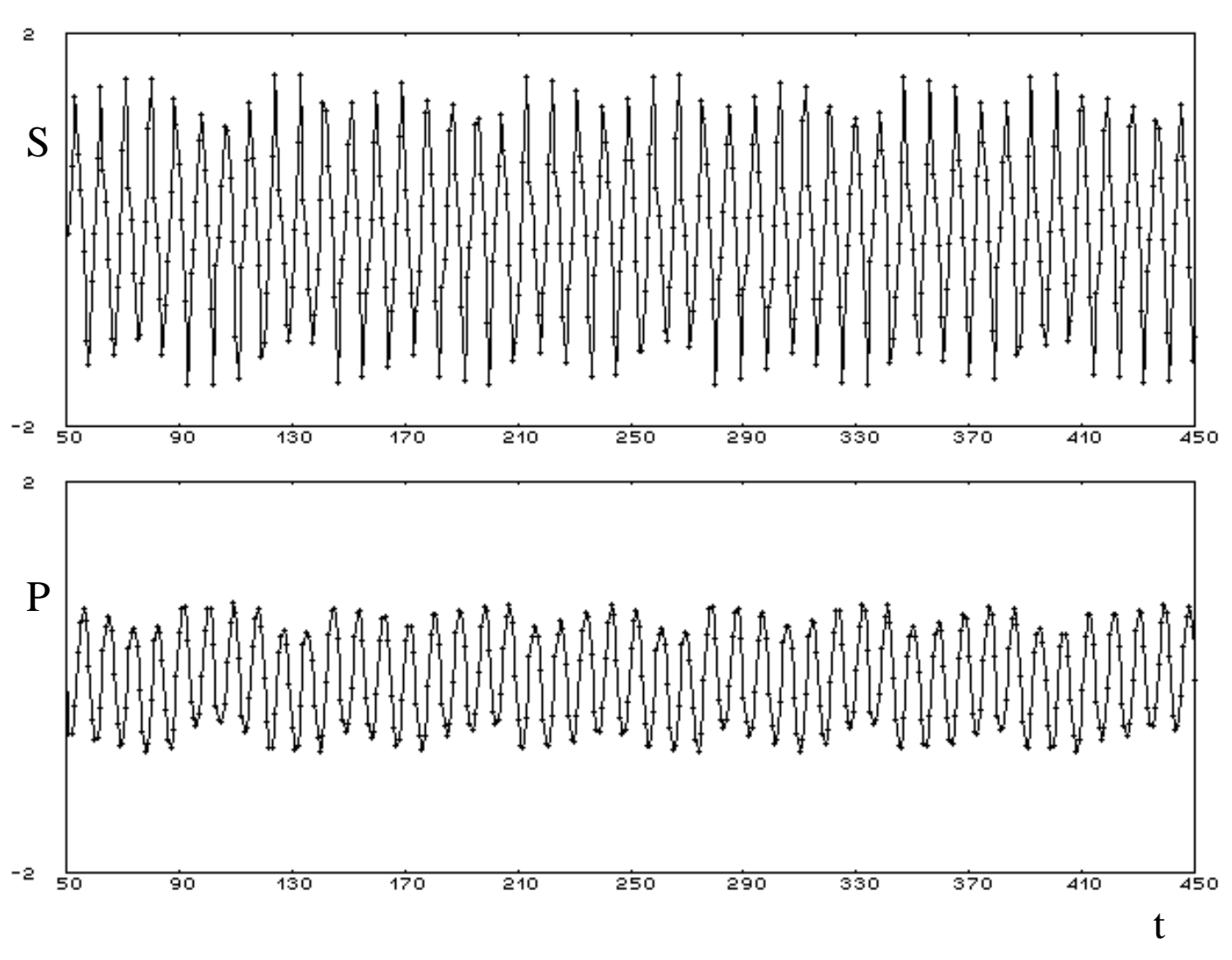

(b)

Fig. 3 


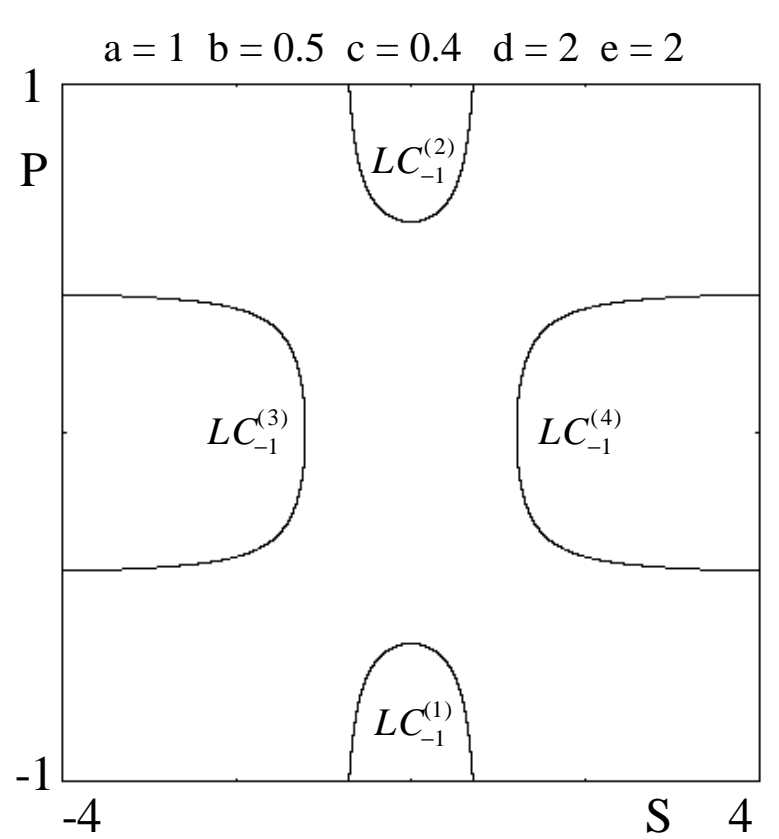

(a)

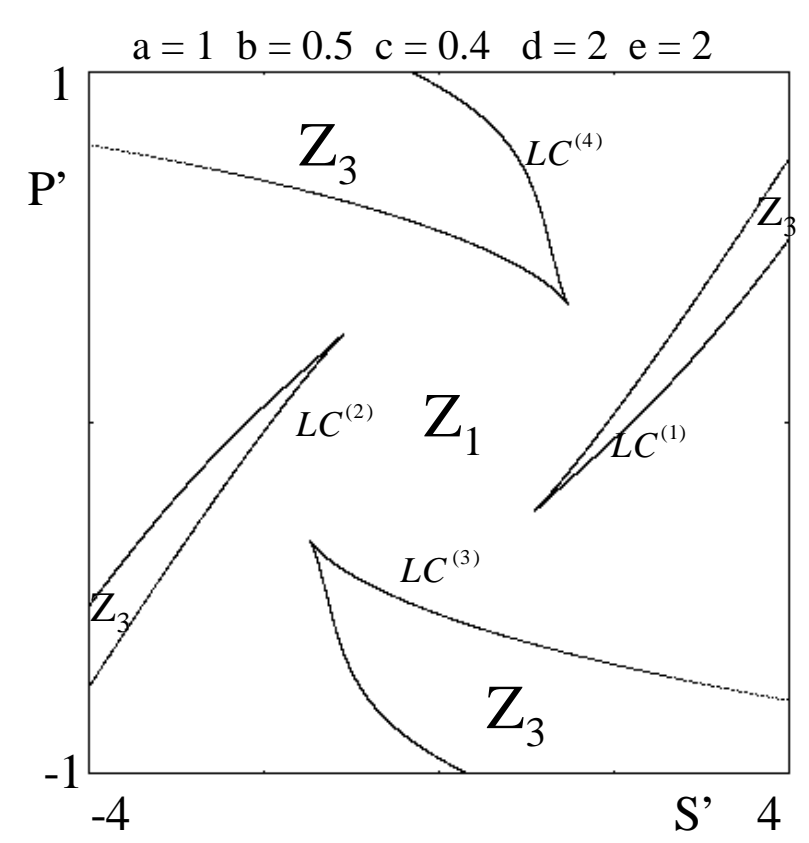

(b)

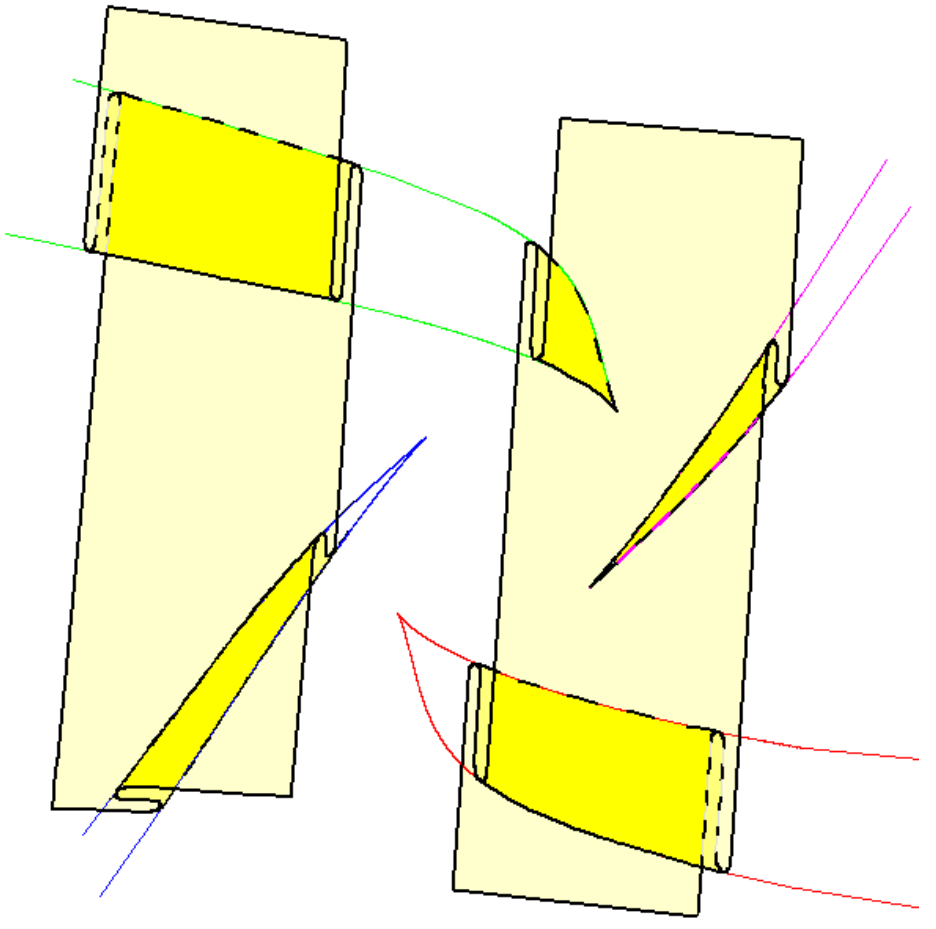

(c)

Fig. 4 


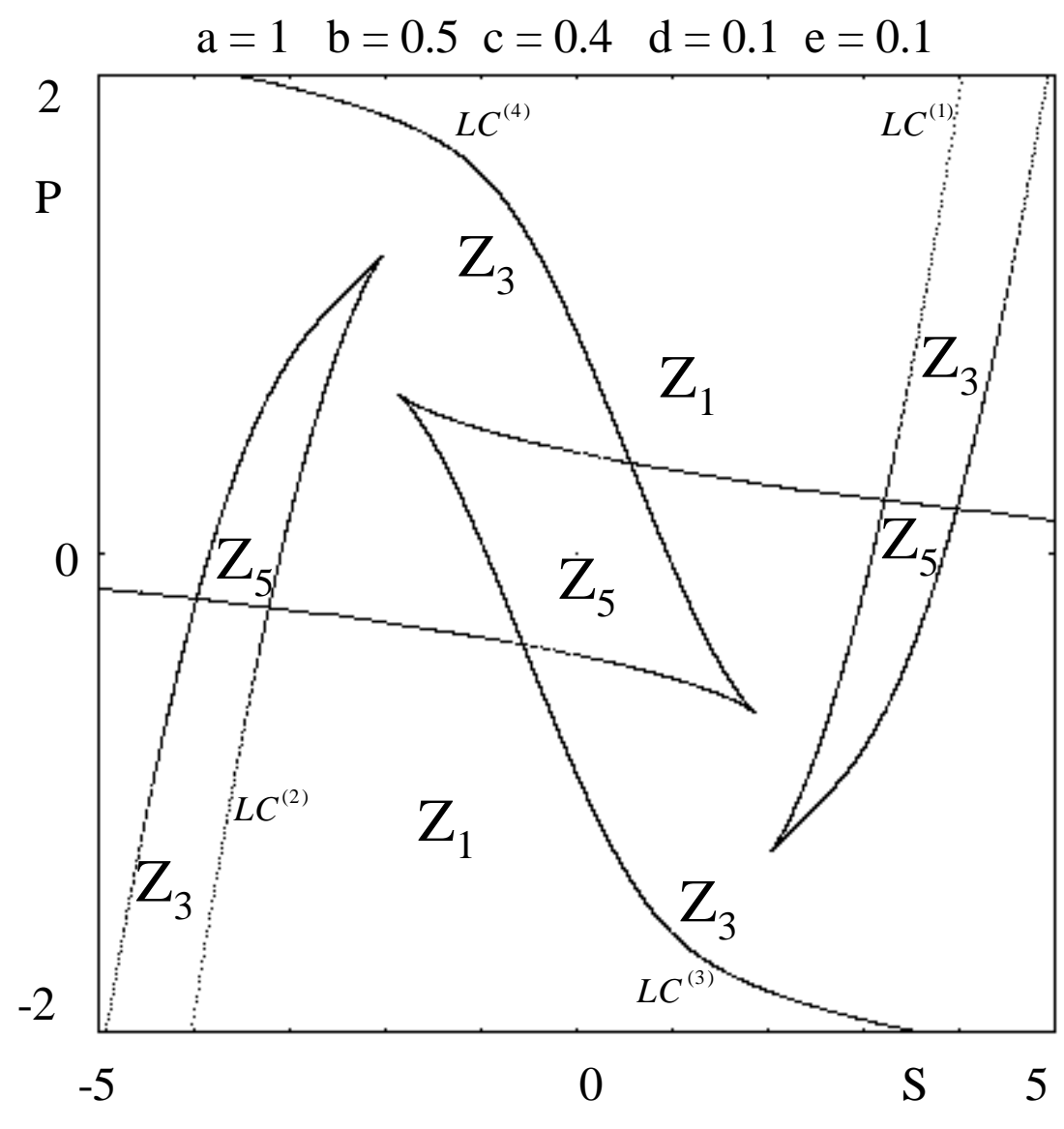

(a)

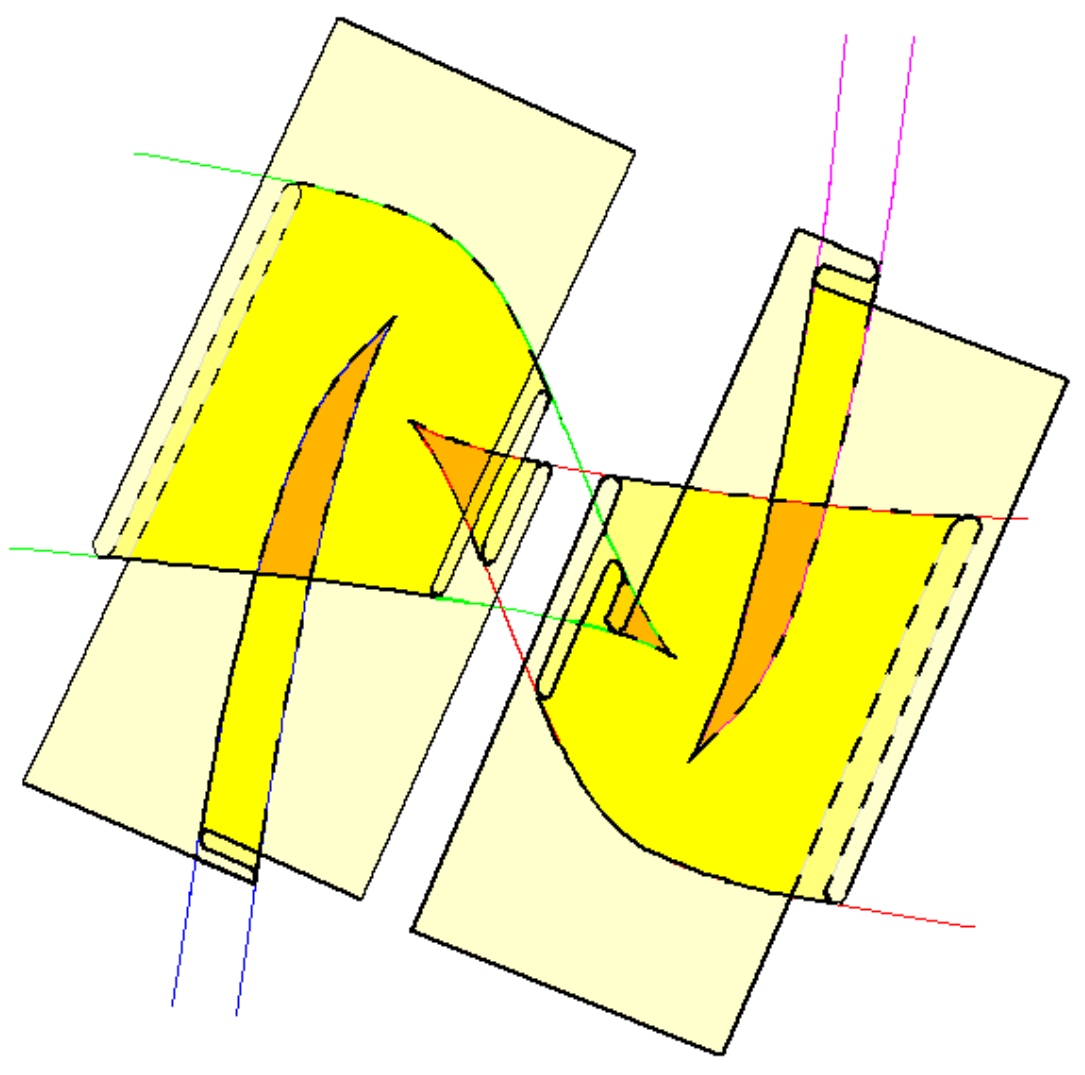

(b)

Fig. 5 


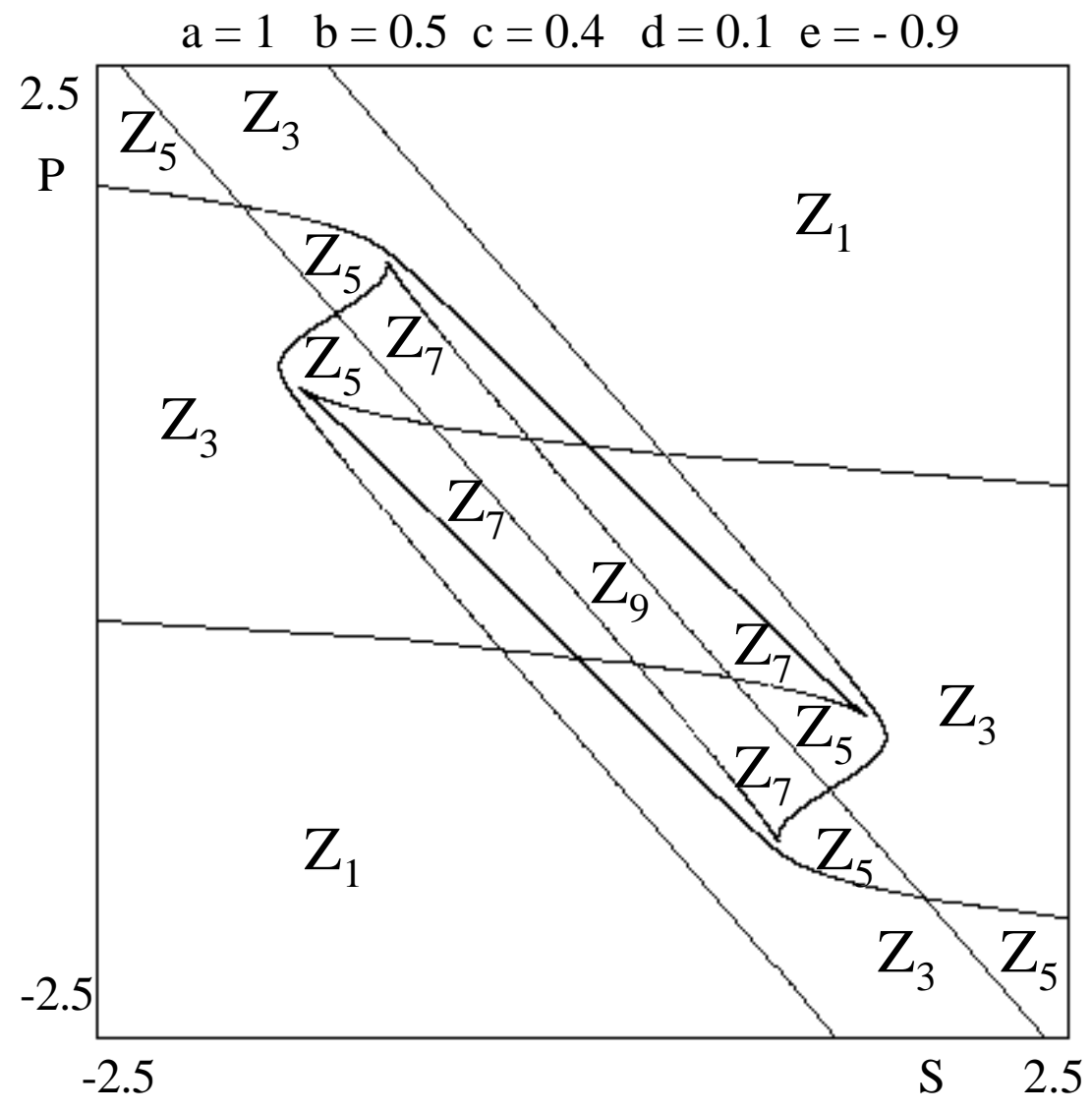

(a)

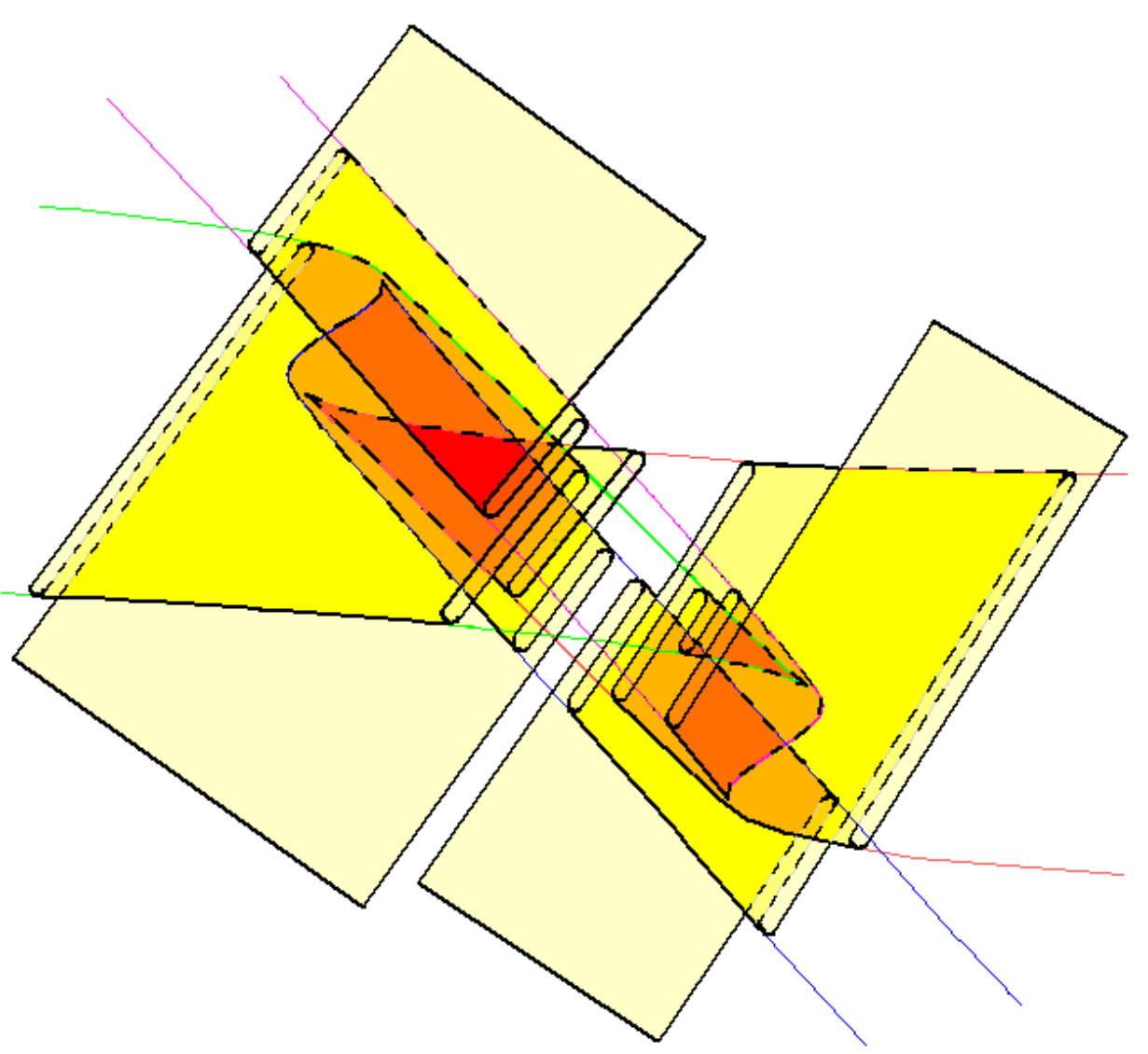

(b)

Fig. 6 

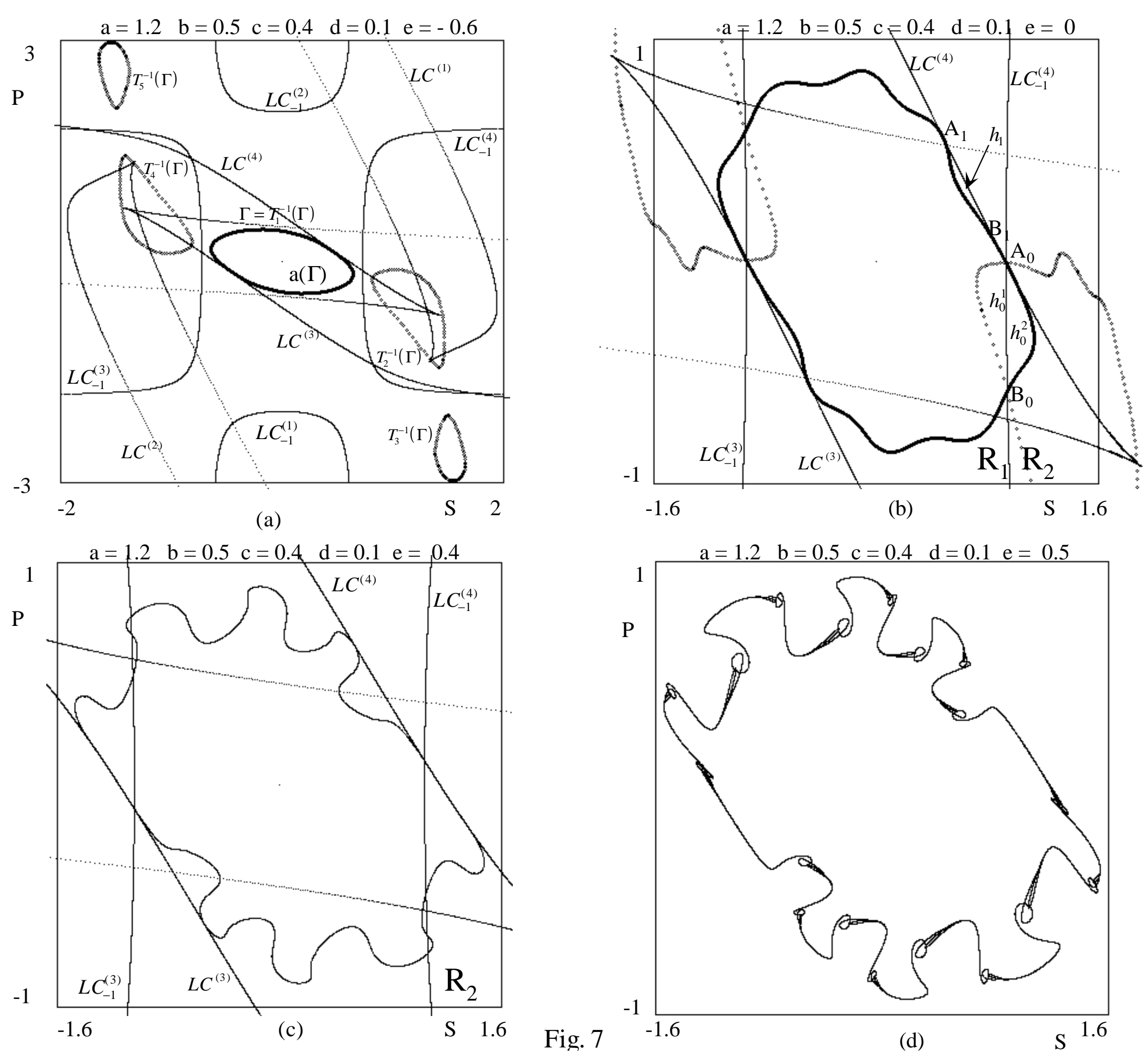


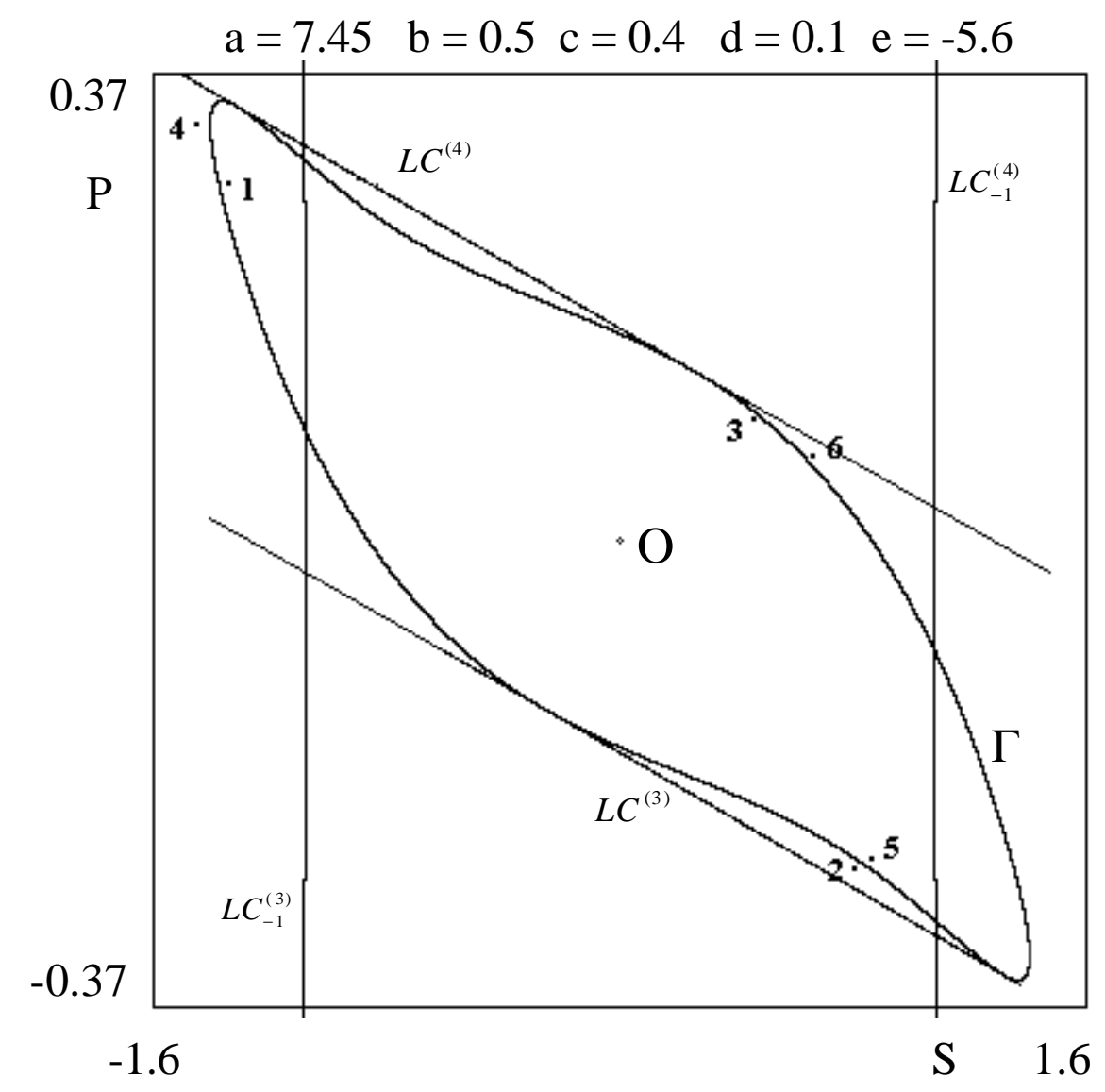

Fig. 8 


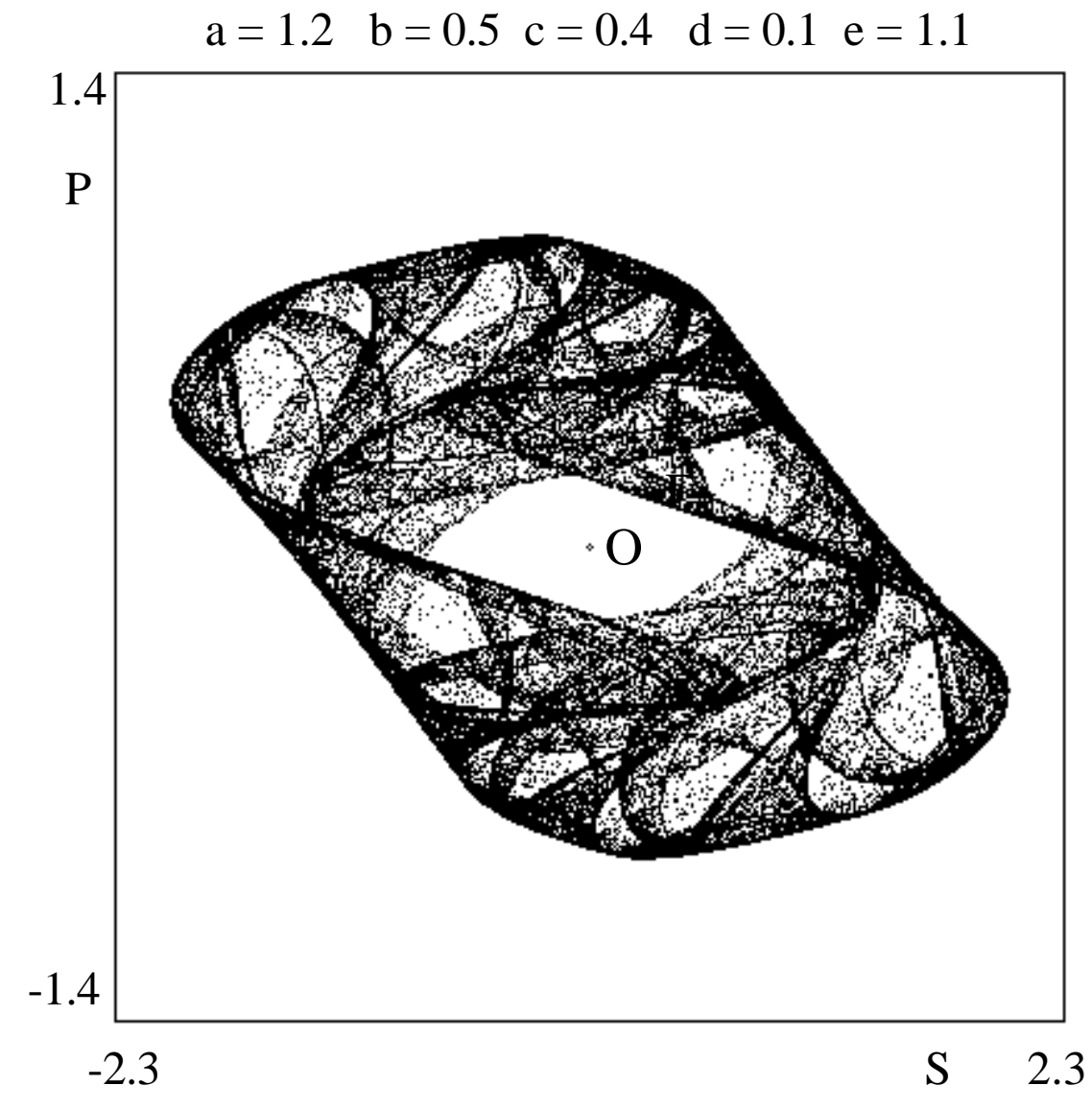

(a)

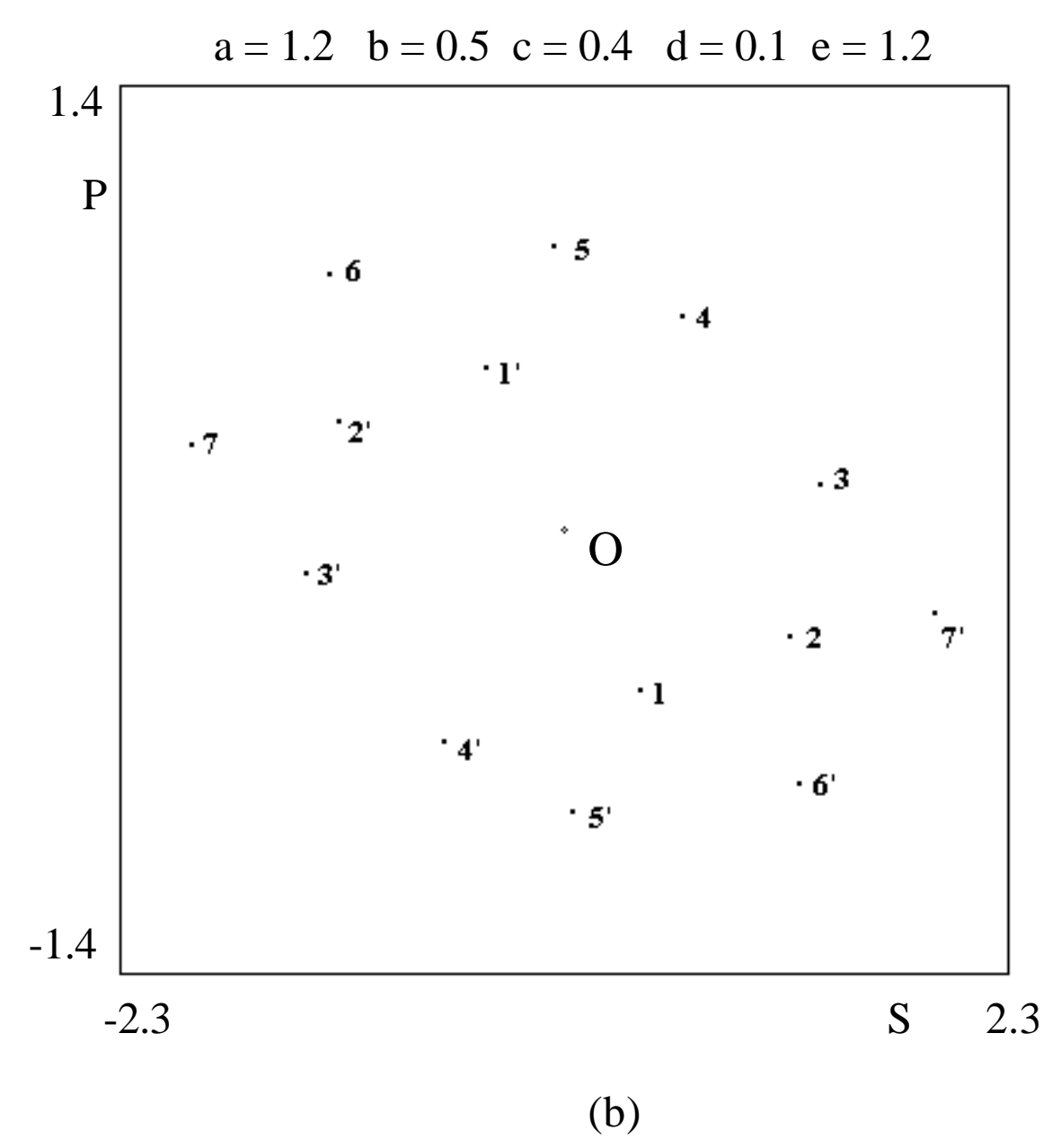

(b) 


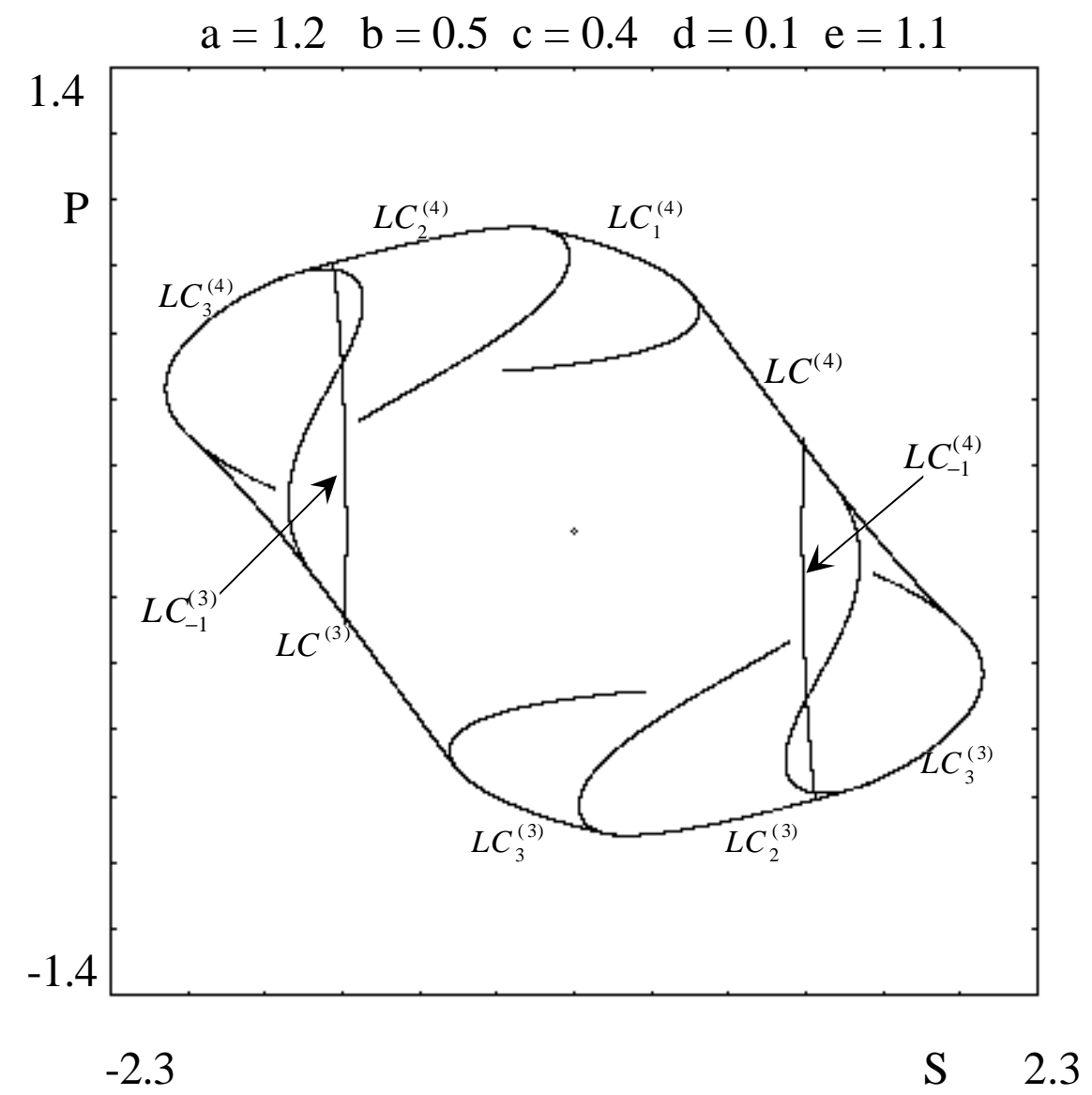

(a)

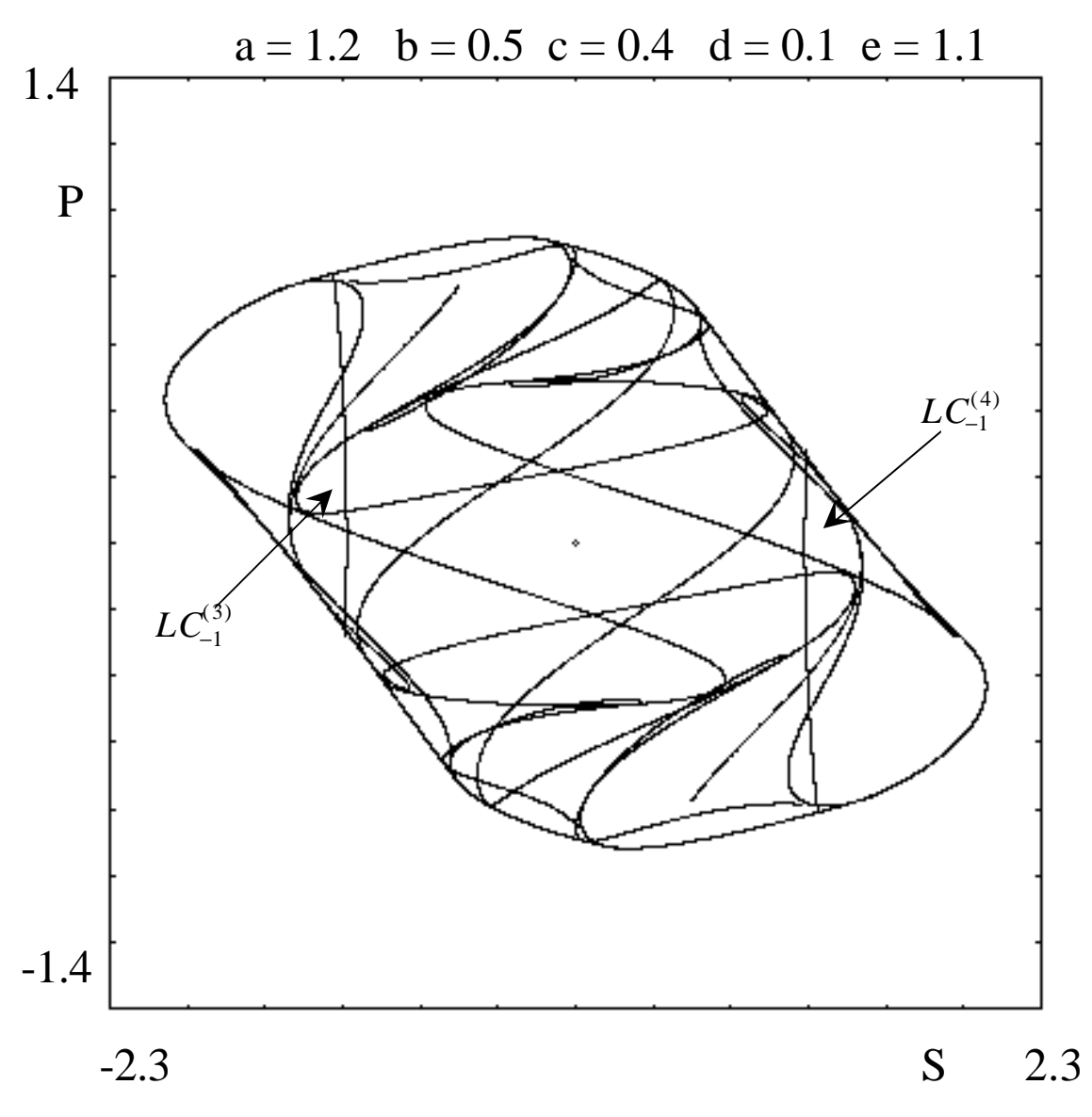

(b)

Fig. 10 

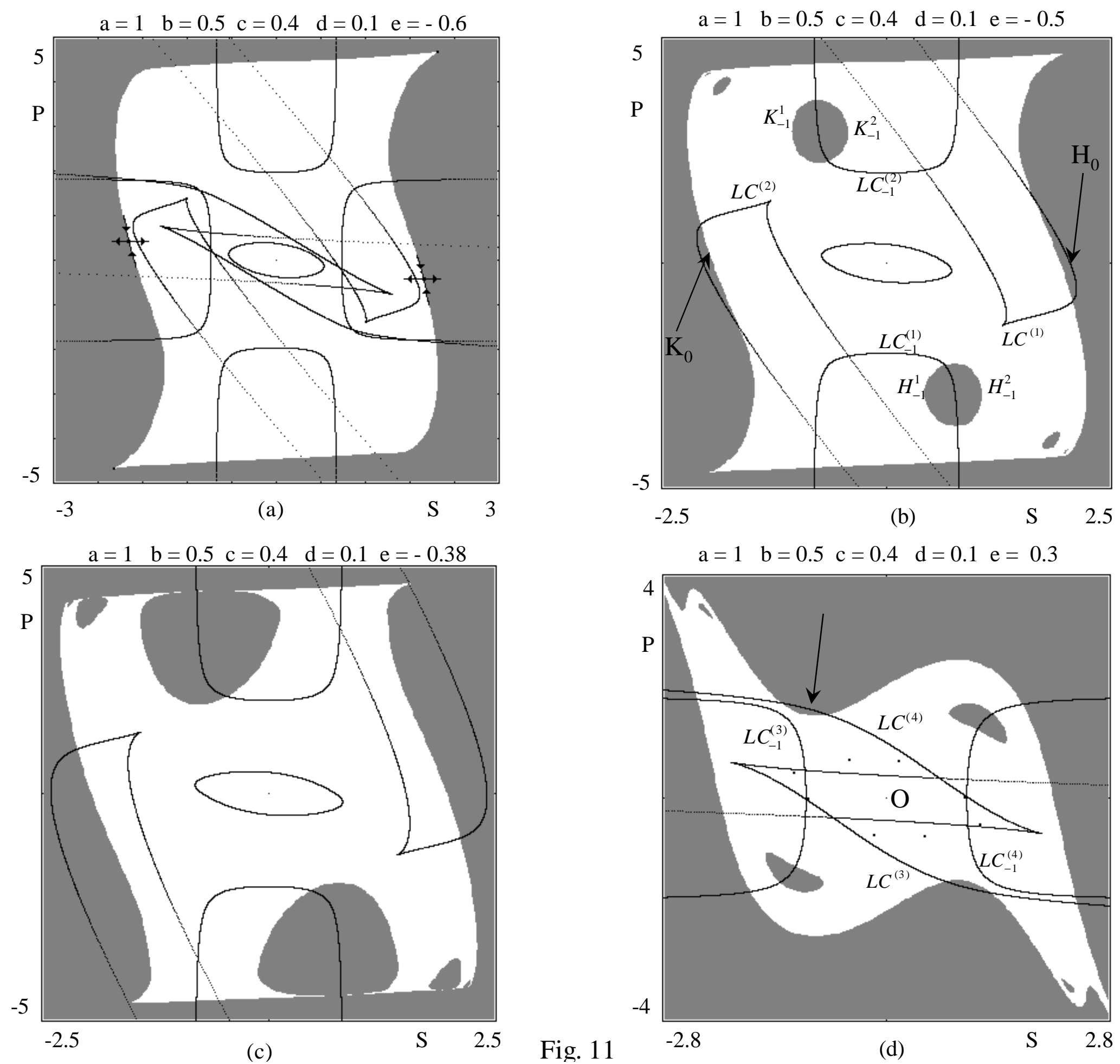

Fig. 11

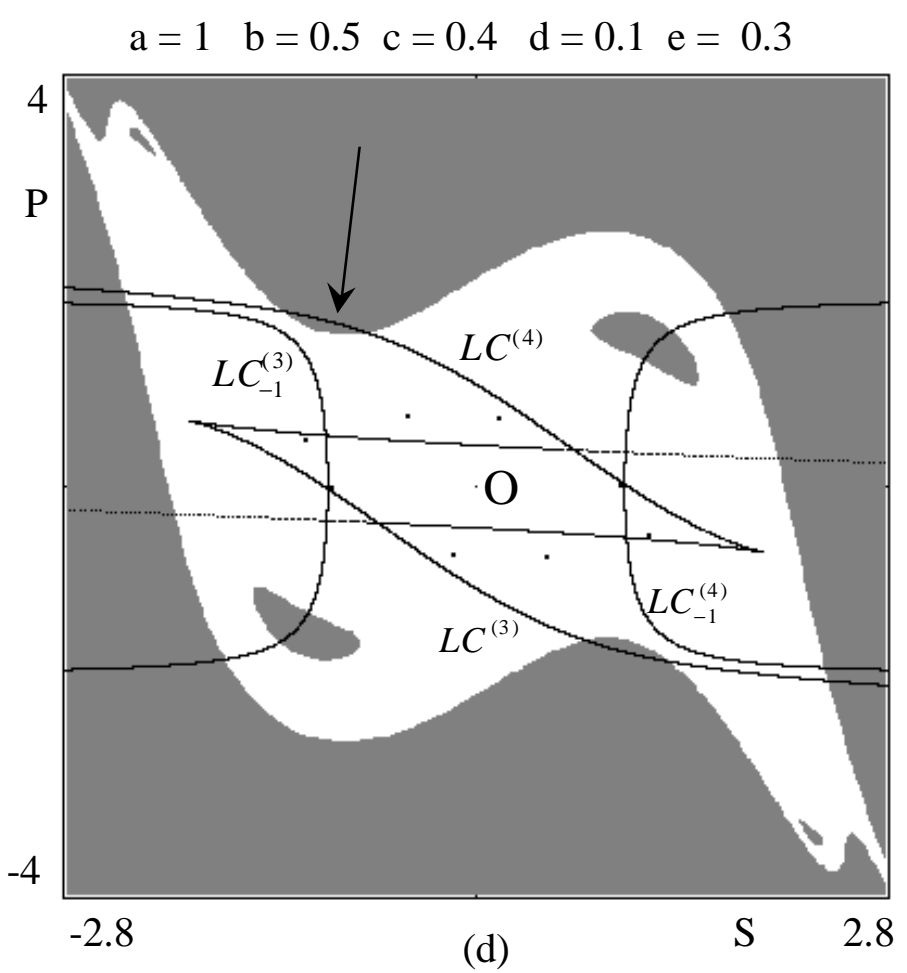




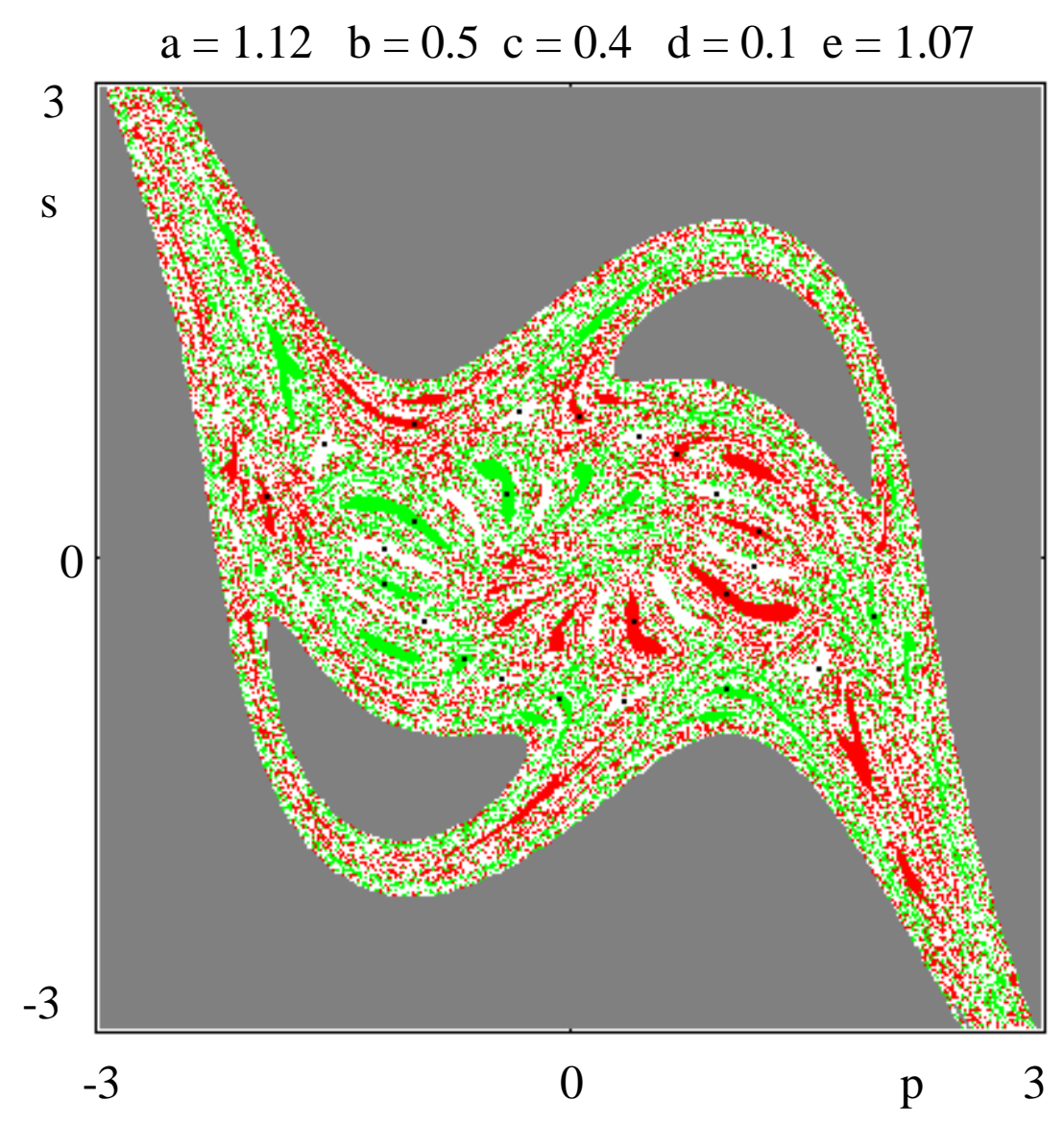

Fig. 12 